\title{
Article
}

\section{Co-formation of the thin and thick discs revealed by APOGEE-DR16 and Gaia-DR2}

Beraldo E silva, Leandro, Debattista, Victor P, Nidever, David, Amarante, Joao A. S. and Garver, Bethany

Available at http://clok.uclan.ac.uk/34790/

Beraldo E silva, Leandro ORCID: 0000-0002-0740-1507, Debattista, Victor P ORCID: 0000-0001-7902-0116, Nidever, David, Amarante, Joao A. S. and Garver, Bethany (2021) Co-formation of the thin and thick discs revealed by APOGEE-DR16 and Gaia-DR2. Monthly Notices of the Royal Astronomical Society, 502 (1). pp. 260-272. ISSN 0035-8711

It is advisable to refer to the publisher's version if you intend to cite from the work. http://dx.doi.org/10.1093/mnras/staa3966

For more information about UCLan's research in this area go to http://www.uclan.ac.uk/researchgroups/ and search for < name of research Group $>$.

For information about Research generally at UCLan please go to http://www.uclan.ac.uk/research/

All outputs in CLoK are protected by Intellectual Property Rights law, including Copyright law. Copyright, IPR and Moral Rights for the works on this site are retained by the individual authors and/or other copyright owners. Terms and conditions for use of this material are defined in the policies page. 


\title{
Co-formation of the thin and thick dises revealed by APOGEE-DR16 and Gaia-DR2
}

\author{
Leandro Beraldo e Silva ${ }^{\circledR},{ }^{\star}$ Victor P. Debattista ${ }^{\circledR}, 1$ David Nidever, ${ }^{2,3}$ João A. S. Amarante ${ }^{\circledR 4,5}$ \\ and Bethany Garver ${ }^{2}$ \\ ${ }^{1}$ Jeremiah Horrocks Institute, University of Central Lancashire, Preston PR1 2HE, UK \\ ${ }^{2}$ Department of Physics, Montana State University, PO Box 173840, Bozeman, MT 59717-3840, USA \\ ${ }^{3}$ NSF's National Optical-Infrared Astronomy Research Laboratory, 950 North Cherry Ave, Tucson, AZ 85719, USA \\ ${ }^{4}$ Key Laboratory for Research in Galaxies and Cosmology, Shanghai Astronomical Observatory, Chinese Academy of Sciences, 80 Nandan Road, Shanghai \\ 200030, China \\ ${ }^{5}$ University of Chinese Academy of Sciences, No.19A Yuquan Road, Beijing 100049, China
}

Accepted 2020 December 16. Received 2020 December 4; in original form 2020 September 4

\begin{abstract}
Since thin disc stars are younger than thick disc stars on average, the thin disc is predicted by some models to start forming after the thick disc had formed, around $10 \mathrm{Gyr}$ ago. Accordingly, no significant old thin disc population should exist. Using 6D coordinates from Gaia-DR2 and age estimates from Sanders \& Das, we select $\sim 24000$ old stars $(\tau>10 \mathrm{Gyr}$, with uncertainties $\lesssim 15$ per cent) within $2 \mathrm{kpc}$ from the Sun (full sample). A cross-match with APOGEE-DR16 ( $\sim 1000$ stars) reveals comparable fractions of old chemically defined thin/thick disc stars. We show that the full sample pericentre radius $\left(r_{\text {per }}\right)$ distribution has three peaks, one associated with the stellar halo and the other two having contributions from the thin/thick discs. Using a high-resolution $N$-body + SPH simulation, we demonstrate that one peak, at $r_{\text {per }} \approx 7.1 \mathrm{kpc}$, is produced by stars from both discs that were born in the inner Galaxy and migrated to the Solar Neighbourhood. In the Solar Neighbourhood, $\sim 1 / 2(\sim 1 / 3)$ of the old thin (thick) disc stars are classified as migrators. Our results suggest that thin/thick discs are affected differently by radial migration inasmuch as they have different eccentricity distributions, regardless of vertical scale heights. We interpret the existence of a significant old thin disc population as evidence for an early co-formation of thin/thick discs, arguing that clump instabilities in the early disc offer a compelling explanation for the observed trends.
\end{abstract}

Key words: Galaxy: abundances-Galaxy: disc-Galaxy: evolution-Galaxy: formation-Galaxy: structure.

\section{INTRODUCTION}

The Milky Way (MW) disc is a compound structure, as first revealed by its double exponential vertical density profile (Yoshii 1982; Gilmore \& Reid 1983). The thin disc is characterized by stars on low-eccentricity orbits and which, on average, are younger than the thick disc. In turn, the thick disc is more centrally concentrated, has a larger fraction of stars on eccentric orbits and is older on average (see e.g. Bovy et al. 2012; Mackereth et al. 2019; Ciucă et al. 2020). Additionally, the MW chemical abundance map $([\alpha / \mathrm{Fe}]$ versus $[\mathrm{Fe} / \mathrm{H}])$ is bi-modal, with $\alpha$-rich stars being more vertically extended, in contrast to $\alpha$-poor stars, and thus associated with the geometrical thick disc (e.g. Adibekyan et al. 2011; Anders et al. 2014; Bensby, Feltzing \& Oey 2014; Nidever et al. 2014). However, the discs defined chemically and geometrically are not identical; in this work, we adopt the chemical definition.

Because the thick disc is old, modelling its formation is intrinsically related to dating the earliest events in the Galaxy's evolution, and several possibilities have been considered, including the early accretion from disrupted satellites (Abadi et al. 2003), heating of a proto-disc by a massive merger (Quinn, Hernquist \& Fullagar

^E-mail: lberaldoesilva@gmail.com
1993; Kazantzidis et al. 2008; Villalobos \& Helmi 2008; Helmi et al. 2018), in situ star formation following a gas-rich merger (Brook et al. 2004), scattering by clumps in an early gas-rich phase (Kroupa 2002; Bournaud, Elmegreen \& Martig 2009; Clarke et al. 2019; Beraldo e Silva et al. 2020), radial migration (Schönrich \& Binney 2009b; Loebman et al. 2011), direct in situ formation from a turbulent thick gas disc (so-called 'upside-down' formation; Bird et al. 2013), mergers with subsequent cosmological inflow of gas along filaments (Agertz et al. 2020; Renaud et al. 2020a,b), and the rapidly changing orientation of the galactic plane (Meng \& Gnedin 2020).

While some of these scenarios (notably the upside-down) predict a sequential formation, with the geometric thin disc significantly forming only after the thick disc, in other scenarios the thin disc starts forming simultaneously with the thick disc, with the consequence that a significant population of old thin disc stars should exist. For instance, Abadi et al. (2003) predict that $\approx 15$ per cent of currently (kinematically defined) thin disc stars should have ages $\tau>10 \mathrm{Gyr}$ in the accretion scenario, while in the major merger picture, the geometric thin remnant should represent 15-25 per cent of the total stellar mass at the end of the merger, according to Villalobos \& Helmi (2008). Additionally, in the clumpy formation scenario, the early disc develops clumps that heat stars vertically (as originally shown by Bournaud et al. 2009) and enrich the medium with $\alpha$-elements, producing a chemical bi-modality (as shown by Clarke et al. 2019) 
and geometric thin + thick discs similar to those observed in the MW (as shown by Beraldo e Silva et al. 2020). Thus, thin + thick disc co-formation and an old thin disc population are natural predictions of this scenario. Other thick disc formation models also suggest a thin + thick co-formation (see e.g. Agertz et al. 2020; Khoperskov et al. 2020; Lian et al. 2020; Renaud et al. 2020a).

Whatever the case for the thin disc's early formation, there is evidence for it growing inside-out at later times (as in, e.g. Chiappini, Matteucci \& Romano 2001; Muñoz-Mateos et al. 2007; Bovy et al. 2016), i.e. stars forming at progressively larger radii, with the consequence that, at a fixed time, stars born at smaller radii are typically metal-richer than those formed in the disc's outer part. Observing these trends is complicated by radial migration (churning), which can move stars inwards or outwards by several kpc on short times. This mechanism changes individual stars angular momenta without changing their radial actions and is more efficient for nearlycircular orbits (Sellwood \& Binney 2002; Roškar et al. 2012). An important practical consequence, due to the density decreasing with radius, is a net movement outward of more metal-rich inner stars, as detected, e.g. in APOGEE (Hayden et al. 2015), LAMOST + RAVE + Gaia-DR1 (Vickers \& Smith 2018), and in simulations (Loebman et al. 2016) - see also Schönrich \& Binney (2009a).

Recently, Prudil et al. (2020) found evidence for a population of 22 RR Lyrae stars (which are older than $10 \mathrm{Gyr}$ - see e.g. Glatt et al. 2008; VandenBerg et al. 2013) with pericentre radii peaking at $r_{\text {per }} \approx$ $7 \mathrm{kpc}$, small vertical excursions $\left(z_{\max }<0.9 \mathrm{kpc}\right)$, high metallicities, and low $\alpha$-abundances, which they deemed a 'conundrum': how can an old population have chemical and kinematic properties typical of the thin disc? And how, in apparent contradiction with an inside-out growth, would this old population be located at such large radii?

In this work, using Sanders \& Das (2018) catalogue, in conjunction with APOGEE-DR16 abundances (Ahumada et al. 2020; Jönsson et al. 2020), we confirm the existence of a significant population of old $(\tau>10 \mathrm{Gyr})$ thin disc stars in the Solar Neighbourhood and a peak in the pericentre radii at $r_{\text {per }} \approx 7 \mathrm{kpc}$ with contributions from both thin and thick discs. We further interpret this old thin disc population as evidence for an early co-formation of the thin and thick discs, with the $r_{\text {per }}$ peak produced by radial migration of both components. In Section 2, we present our data sample and explain the orbit integration procedure. In Section 3, we present our results supporting an early co-formation of the thin and thick discs. The location of the $r_{\text {per }}$ peak is investigated with an $N$-body $+\mathrm{SPH}$ simulation in Section 4, where we show that these stars must have formed in the inner disc and end up at larger radii due to radial migration. In Section 5, we discuss the implications of this finding for different scenarios of the early evolution of the MW and, and in particular, for the thick disc. We also discuss the differences of radial migration in the thin and thick discs and how our results solve the apparent conundrum of thin disc RR Lyrae stars. We summarize our conclusions in Section 6.

\section{OBSERVATIONAL DATA AND ORBIT INTEGRATION}

We explore the astrometric data from Gaia-DR2 and age estimates from the value-added catalogue of Sanders \& Das (2018). This catalogue is based on several spectroscopic surveys data and provides distance, mass and age estimates for $\sim 3$ million stars. It also provides Galactocentric cylindrical coordinates and velocities and, when available, IDs of stars identified in the surveys. We refer the reader to the original paper for details.
We select stars in the subsamples of giants (defined as $\log g>$ 3 dex and $\log _{10}\left(T_{\text {eff }} / K\right)<3.73$ ), and turnoff stars (defined as $3.6<$ $\log g<4.5$ and $\left.\log _{10}\left(T_{\text {eff }} / K\right)<4.1\right)$, for which ages are most reliable, according to Sanders \& Das (2018). We select old stars ( $\tau>10 \mathrm{Gyr}$ ), with uncertainties $\sigma_{\tau}<1.5 \mathrm{Gyr}$, within a distance $d<2 \mathrm{kpc}$ from the Sun. Additionally, we require accurate parallaxes $\left(\left|\varpi / \sigma_{\varpi}\right|>\right.$ $5)$ and line-of-sight velocities $\left(\left|v_{\text {los }} / \sigma_{v_{\text {los }}}\right|>5\right)$. Note that this last requirement suppresses stars with small $v_{\text {los }}$, even if $\sigma_{v_{\text {los }}}$ is as small as $1 \mathrm{~km} \mathrm{~s}^{-1}$, mainly suppressing the contribution of near-by thin disc stars. Thus, this is a conservative cut for the detection of a significant old thin disc population and does not affect our conclusions. In order to avoid stars with too small age uncertainties, artificially produced by the model isochrone gridding, we require $\sigma_{\log _{10} \tau}>0.015 \mathrm{dex}$, as suggested by Sanders \& Das (2018). Finally, we restrict to stars with the flag best $=1$ (stars with a match in Gaia and no duplicate observations or observational problem detected). This results in a sample of 23857 stars, with a median $\sigma_{\tau} \approx 0.99 \mathrm{Gyr}$ (and maximum $\sigma_{\tau}=1.5 \mathrm{Gyr}$, defined in the quality cut).

We use the phase-space coordinates of our sample as initial conditions and, with the help of the AGAMA package (Vasiliev 2019), integrate the orbits in the Galactic potential of McMillan (2017). We identify 62 unbounded stars, which we exclude, leaving a sample with 23795 stars. Each orbit is integrated forwards for 10 dynamical times (i.e. for $\sim 2 \mathrm{Gyr}$ ), and we calculate the maximum height from the Galactic plane, $z_{\max }$, and the pericentre and apocentre radii, $r_{\text {per }}$ and $r_{\text {apo }}$, respectively. The eccentricity is estimated as

$e=\frac{r_{\text {apo }}-r_{\text {per }}}{r_{\text {apo }}+r_{\text {per }}}$.

When further splitting the data into thick and thin discs, we crossmatch with APOGEE-DR16 (Ahumada et al. 2020; Jönsson et al. 2020 ), resulting in a sample of $\sim 1000$ stars with accurate metallicities and $\alpha$-abundances. Rojas-Arriagada et al. (2019) showed that magnesium is the $\alpha$-element with most reliable abundance estimates in APOGEE and we therefore use $[\mathrm{Mg} / \mathrm{Fe}]$ to trace the $\alpha$-abundances.

\section{OBSERVATIONAL RESULTS}

We now show the results obtained from our sample of old stars in the Solar Neighbourhood. We start analysing the distribution of eccentricities and pericentre radii, demonstrating the presence of three peaks, one of which is at $r_{\text {per }} \approx 7 \mathrm{kpc}$. In Section 3.2, we cross-match the data with the APOGEE-DR16, split the resulting sample into chemical thin and thick discs, and investigate the chemo-kinematic properties of these components, showing their relative contributions to the $r_{\text {per }}$ peaks. In Section 3.3, we present the first evidence that the peak at $r_{\text {per }} \approx 7 \mathrm{kpc}$ is produced by radial migration. This is further investigated with a high-resolution simulation in Section 4.

\subsection{Peaks in pericentre radius}

Fig. 1, upper panel, shows contours for eccentricity versus pericentre radius for our sample. The dashed lines represent two fixed values of apocentre radius $r_{\text {apo }}$ - see equation (1). The absence of data with $r_{\text {apo }}<6 \mathrm{kpc}$ reflects the selection of stars within $d<2 \mathrm{kpc}$ from the Sun, given the Solar position $R_{\odot} \approx 8 \mathrm{kpc}$, while the vast majority of stars have $r_{\text {apo }}<14 \mathrm{kpc}$. The distribution peaks along a line of approximately constant $r_{\text {apo }}$, in $8 \lesssim r_{\text {apo }} / \mathrm{kpc} \lesssim 9$, which also must be a selection effect, given the Solar position and the larger time stars spend near their apocentres. The contours suggest the presence of three peaks: the first at $\left(r_{\mathrm{per}} / \mathrm{kpc}, e\right) \approx(0.5,0.9)$, the second at $\approx(5-6,0.25)$, and the third at $\approx(7,0.1)$. 

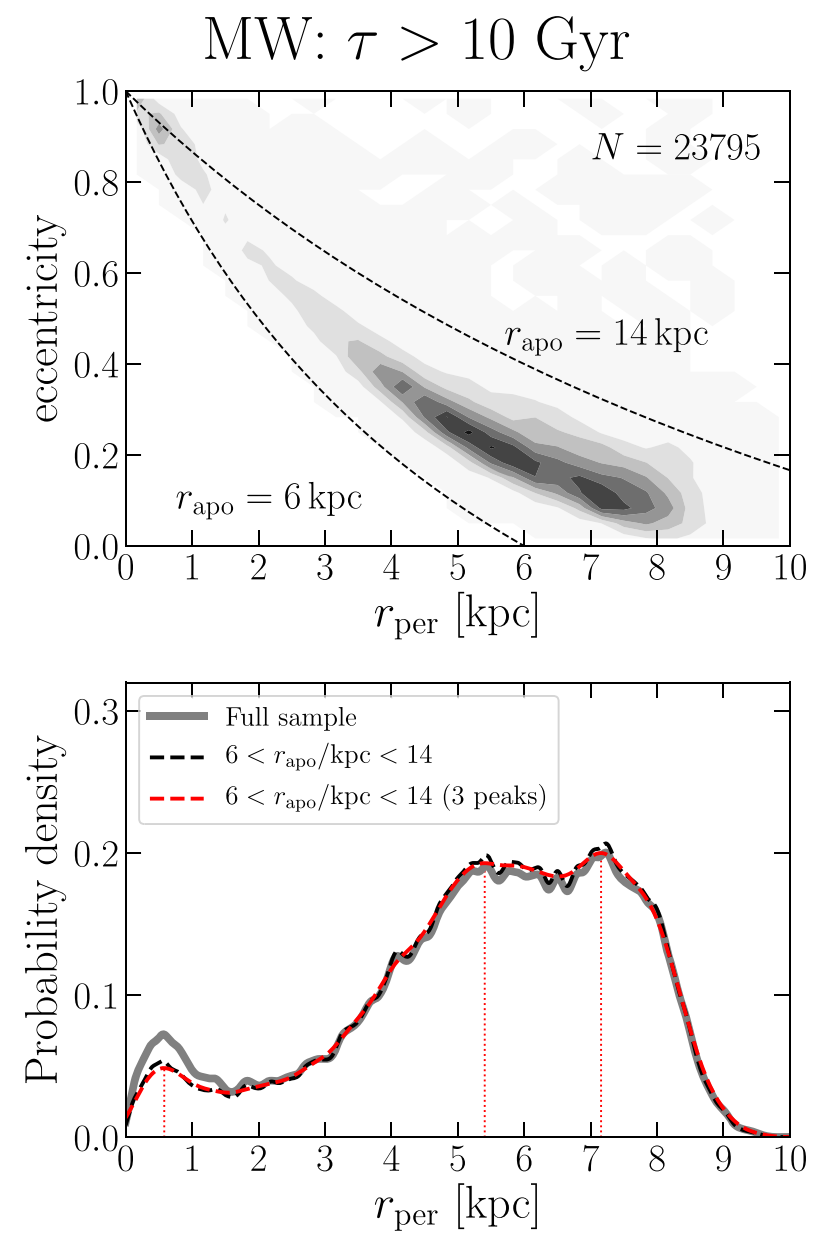

Figure 1. Top panel: distribution of old (turnoff + giant) stars in eccentricity versus pericentric radius. Ages and astrometric data from Sanders \& Das (2018). Bottom panel: KDEs of $r_{\text {per }}$ : for the full sample (grey), for $6<$ $r_{\text {apo }} / \mathrm{kpc}<14$ (black dashed) and for this region and the most detailed KDE producing three peaks (dashed red) at the positions shown by vertical lines (see the text for details). A statistical test rules out models with less than three peaks.

The lower panel shows the kernel density estimate (KDE) of $r_{\text {per }}$ (i.e. of the projection of the upper panel on the $x$-axis) using a Gaussian kernel with window width $h=0.07$, which was chosen by cross-validation (solid grey), where the three peaks mentioned before can be seen more clearly. In order to eliminate stars with extreme or unrealistic kinematic properties, which can arise because of the assumed model for the MW's potential, we select stars with $6<$ $r_{\text {apo }} / \mathrm{kpc}<14$, i.e. between the dashed curves in the upper panel. The $r_{\text {per }} \mathrm{KDE}$ for stars satisfying this cut (black dashed in the lower panel) is very similar to that of the total sample, with the only noticeable difference being a suppression of high-eccentricity orbits in the first peak, not relevant for our analysis. Thus, in the rest of the paper we use this restricted sample $(N=22776$ stars $)$.

In order to check for the real significance of the peaks, as opposed to random fluctuations, we perform the test proposed by Silverman (1981) - see Appendix A. The presence of (at least) three peaks is confirmed with high statistical significance. This confirms the detection of three peaks by Prudil et al. (2020) with a restricted sample of 314 RR Lyrae stars (under the assumption of Gaussian components, not assumed in our analysis). This shows that this $r_{\text {per }}$ distribution is not associated with any specific feature of their sample, but indicates the presence of distinct Galactic components.

The red dashed curve in Fig. 1 (lower panel) is the $r_{\text {per }} \mathrm{KDE}$ obtained with the $h_{\text {crit }}$ for three peaks from Table A1, i.e. the most detailed KDE having no more than three peaks. The only, barely noticeable, difference with respect to the black curve is the smoothing of the little wiggles. The three peaks are located at $r_{\text {per }} \approx 0.59,5.4$, and $7.14 \mathrm{kpc}$ (as indicated by the vertical red dotted lines).

Next, we analyse the chemical and kinematic properties of stars in these three populations and use the results to suggest their association with known structures in the MW.

\subsection{Halo, thick and thin discs}

The catalogue of Sanders \& Das (2018) provides ages, 6D phasespace coordinates and metallicities, but lacks information on the $\alpha$-abundances. For this, we cross-match the sample with APOGEEDR16, which results in 1049 (turnoff + giant) stars. The chemical abundances $([\mathrm{Mg} / \mathrm{Fe}]$ and $[\mathrm{Fe} / \mathrm{H}])$ for these stars from APOGEEDR16 are shown in Fig. 2 (upper panel). Colours represent the different populations represented by the separating straight lines (defined by eye, similarly to the definitions of e.g. Adibekyan et al. 2011; Mackereth et al. 2019), which define regions for the stellar halo (yellow), thick disc (red), and thin disc (blue). The black point at the bottom left-hand corner at the upper panel illustrates the median uncertainties in $[\mathrm{Mg} / \mathrm{Fe}]$ and $[\mathrm{Fe} / \mathrm{H}]$, as reported in APOGEE-DR16. The lower panel shows the distribution of these stars in the $e$ versus $r_{\text {per }}$ plot. In the two panels, point sizes are proportional to $z_{\max }$.

The most metal-poor and generally $\alpha$-rich stars (yellow points) occupy the locus, in the chemical map, identified with the halo. These stars are typically clustered around small $r_{\text {per }}$ and high $e$, and have large vertical excursions, with $z_{\max }$ reaching $\gtrsim 10 \mathrm{kpc}$, consistent with the assignment to the stellar halo, with a probable accreted origin - see Belokurov et al. (2018) and Helmi et al. (2018). The red points represent stars with typical thick disc chemical abundances, i.e. they are $\alpha$-rich and reasonably metal-poor. These stars spread across all values of $e$ and $r_{\mathrm{per}}$ in the lower panel, but seem to cluster around $r_{\text {per }} \approx 5 \mathrm{kpc}$ and $e \approx 0.25$. Additionally, these stars have $z_{\max }$ as large as a few kpc, which is consistent with their classification as thick disc stars. Finally, the $\alpha$-poor, metal-rich stars (blue points) spread around $4 \lesssim r_{\text {per }} / \mathrm{kpc} \lesssim 8$ and $e \approx 0.2$ and typically have small vertical excursions $z_{\max }$, compatible with the thin disc.

At first sight the features just listed could suggest the identification, from left to right in Fig. 1, of the first $r_{\text {per }}$ peak with halo stars, the second with the thick disc and the third with the thin disc. However, a closer inspection shows that both the thin and thick disc components contribute distinctively to each of the second and third $r_{\text {per }}$ peaks, as we show in Section 3.3. Whatever the case may be, the identification of three peaks, seemingly associated to the halo, thin, and thick discs, agrees with the results from Prudil et al. (2020), although the position of the second peak differs from the value $r_{\text {per }} \approx 3 \mathrm{kpc}$ found in that work. This reinforces their conclusion about the third peak detection and its main relation to an old thin disc population, while showing that it is not associated with any specific feature of their sample, but it represents a significant Galactic component.

Our separation in the chemical space shown in Fig. 2 results in $\sim 17$ per cent of the stars in the halo, $\sim 54$ per cent in the thick disc, and $\sim 29$ per cent in the thin disc. However, these fractions should not be considered as precisely describing the whole Galaxy, since precise fractions would require a detailed analysis of the APOGEEDR16 selection function, which is beyond the scope of this work. In Appendix B, we apply different simple geometric and age cuts and 


\section{APOGEE-DR16: $\tau>10$ Gyr}
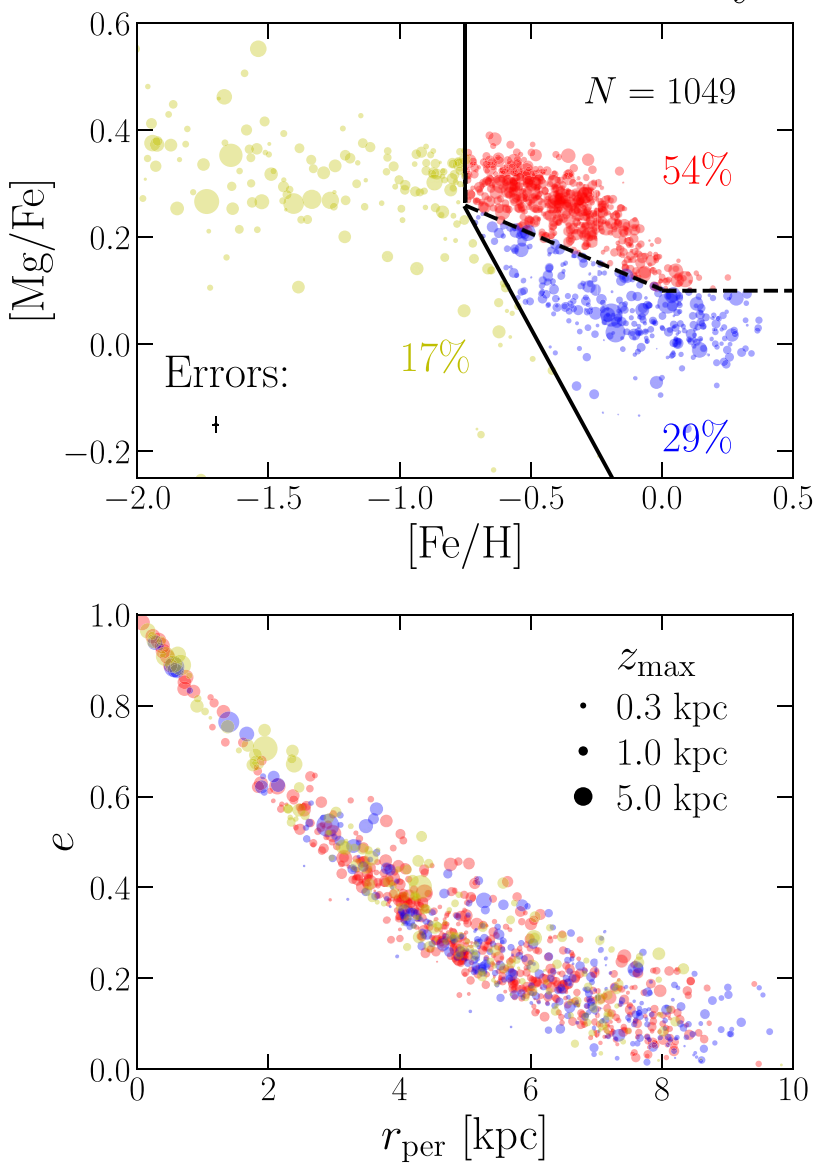

Figure 2. Chemical map (top panel) and eccentricity, $e$, versus pericentre radius, $r_{\text {per }}$, distribution (bottom panel), for the cross-match with APOGEEDR16. Colours define halo (yellow), thick (red), and thin (blue) disc stars, split through the straight lines, with fractions shown in the top panel. Point sizes are proportional to $z_{\max }$. The black point in the top panel shows the median uncertainties ('errors') in [Fe/H] and [Mg/Fe]. Considering the peaks in Fig. 1, the first (from the left- to right-hand side) is predominantly associated to the halo $\left(\alpha\right.$-rich, very metal-poor, and high $\left.z_{\max }\right)$, while the second and third have significant contributions from the thick disc ( $\alpha$-rich, reasonably metal-poor, and intermediate $z_{\max }$ ) and the thin disc ( $\alpha$-poor, metal-rich, and small $z_{\max }$ ). The top panel suggests that the thin/thick discs were co-forming in the MW's very first Gyr, with comparable star counts.

show that the fractions of old thin and thick disc stars typically vary around $\sim 20-30$ and $\sim 40-55$ per cent, respectively - see Table B1.

Most importantly, since $[\mathrm{Fe} / \mathrm{H}]$ and $[\mathrm{Mg} / \mathrm{Fe}]$ are conserved over a star's lifetime, Fig. 2 shows that in the very first Gyr of the MW's evolution, not only the thick disc but both chemical thin and thick discs were forming, with comparable star counts. The conclusion is similar, with poorer statistics, if we select stars older than $11 \mathrm{Gyr}$. In this case, the cross-match with APOGEE-DR16 results in 573 stars (with median age uncertainty of $\approx 0.84 \mathrm{Gyr}$ ). Of these, 21 per cent belong to the halo, 54 per cent to the thick disc, and 25 per cent to the thin disc - see Table B1. In Section 5, we develop this conclusion and discuss the implications for various thick disc formation scenarios.

We now focus on the thin and thick discs. When referring to the $r_{\text {per }}$ peaks (Fig. 1, lower panel), we neglect the left-hand peak associated to the stellar halo, and call the remaining peaks, at $r_{\text {per }} \approx 5.4$ and $\approx 7.14 \mathrm{kpc}$, the low and high $r_{\text {per }}$ peaks, respectively.

\subsection{Migrators and non-migrators in the thin and thick discs}

The solid lines in Fig. 3 show the KDEs of chemical and kinematic quantities for the chemically defined thick disc (upper panel, 570 stars) and thin disc (lower panel, 305 stars). The thin disc $r_{\text {per }}$ distribution has two peaks, one at $r_{\text {per }} \approx 5 \mathrm{kpc}$ and another at $r_{\text {per }} \approx 7 \mathrm{kpc}$, while the thick disc peaks at $r_{\text {per }} \approx 5 \mathrm{kpc}$ and has a bump at $r_{\text {per }} \approx 7 \mathrm{kpc}$. This shows that the low and high $r_{\text {per }}$ peaks (second and third, in Fig. 1) have distinct contributions from both the thin and thick discs, although the thick disc contributes mostly to the low $r_{\text {per }}$ peak, while the thin disc gives similar contributions to the low and high $r_{\text {per }}$ peaks. This contrasts with the conclusions of Prudil et al. (2020), who tentatively attribute the low and high $r_{\text {per }}$ peaks to the thick and thin discs, respectively.

Using a Gaussian Mixture Model (GMM; Pedregosa et al. 2011), we split the thin and thick discs $r_{\text {per }}$ distributions into three Gaussians each, although only showing the two relevant components (the third Gaussian, not shown, lies at small $r_{\text {per }}$ and large $e$ and is probably due to contamination from the halo). These Gaussian components are shown as dashed and dotted lines in the first column of Fig. 3. With these Gaussians, we define two subsamples, according to the highest probability of belonging to one or the other Gaussian component. In the other columns, the dashed and dotted lines represent the KDEs of the corresponding quantities for these fixed subsamples.

The second column shows that the thin disc eccentricity distribution peaks at $e \approx 0.2$ and extends to $e \lesssim 0.7$, while the thick disc peaks at $e \approx 0.25$ and is slightly broader. For both discs, the high (low) $r_{\text {per }}$ subsample is characterized by lower (higher) eccentricities. In principle, this could be seen as a simple manifestation of the anticorrelation between $r_{\text {per }}$ and eccentricity expressed by equation (1) and shown in Figs 1 and 2. However, the $r_{\text {per }}$ peaks and bumps (in both discs) indicate the existence of a distinct population with $r_{\mathrm{per}} \approx 7.1 \mathrm{kpc}$, which is characterized by nearly-circular orbits.

The KDEs for $[\mathrm{Fe} / \mathrm{H}]$ and $[\mathrm{Mg} / \mathrm{Fe}]$ (third and fourth columns) show that, for both the thin and thick discs, the two Gaussian $r_{\text {per }}$ components (dashed and dotted curves) have chemical distributions similar to those of the total samples (solid lines), thus indicating that the $r_{\text {per }}$ subsamples are typical chemical thin or thick disc samples, and not due to cross-contamination.

The simulation-based results of Section 4.3 make clear that the high $r_{\text {per }}$ peak is produced by stars which formed at inner radii and moved to the current location via radial migration (churning), while the low $r_{\text {per }}$ peak is produced by non-migrators. However, hints for this can be anticipated already using simple arguments. For simplicity, we focus on the thin disc (bottom row in Fig. 3): first, since the low and high $r_{\text {per }}$ subsamples (dashed and dotted lines, respectively) have similar ages ( $\tau>10 \mathrm{Gyr}$ ), their similar chemical abundances suggest that they must have been born at similar radii. Stars born at the same radius but in orbits with different eccentricities would have different $r_{\text {per }}$ but, instead of producing peaks or bumps (as observed in Figs 1 and 3), a continuous (i.e. unimodal) distribution would be expected in the $r_{\text {per }}$ distribution, if migration was not taking place. Secondly, the metallicity distribution extends to (a priori) high values for the outer radii, which was shown by Loebman et al. (2016) to be produced by radial migration of stars born at inner radii. Thirdly, the high $r_{\text {per }}$ peak is associated with stars in low-eccentricity orbits, for which radial migration is most effective (Sellwood \& Binney 2002; Roškar et al. 2012). Furthermore, radial migration does not heat the orbits, i.e. it does not increase their eccentricities. This is compatible with the idea that stars contributing to the high $r_{\text {per }}$ peak migrated to their current location while preserving low eccentricities. Therefore, we anticipate the simulation-based results of Section 4.3 

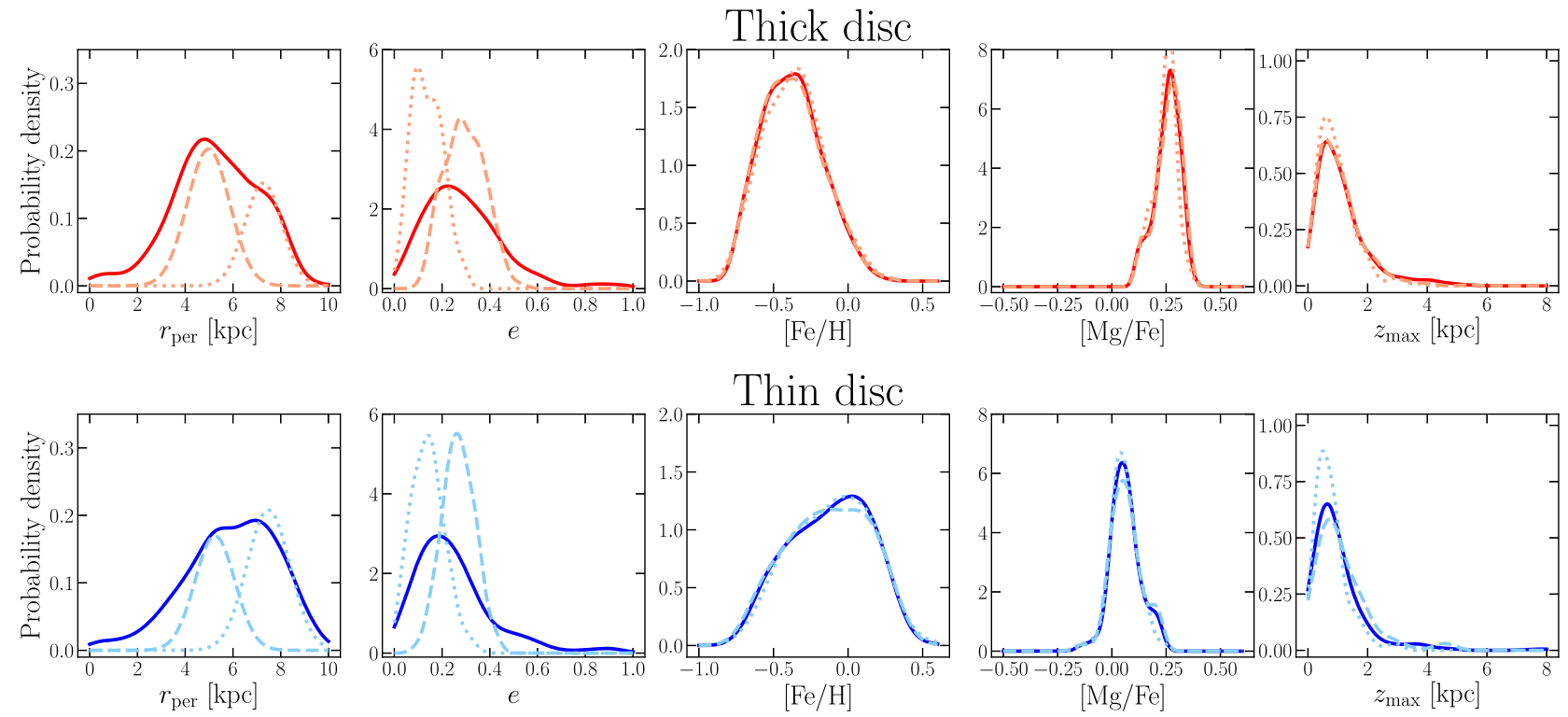

Figure 3. KDEs for kinematic quantities and chemical abundances for the thin disc ( 305 stars) and thick disc (570 stars) from APOGEE-DR16. The $r_{\text {per }}$ distribution has two peaks/bumps for both discs, which we model as two Gaussians, shown in the first column as dotted and dashed curves. In the other columns, dotted and dashed curves show the KDEs of the respective quantities for these two subsamples. The larger $r_{\text {per }}$ subsamples (dotted) are characterized by lower eccentricities, while the lower $r_{\text {per }}$ subsamples (dashed) by higher eccentricities. The $[\mathrm{Fe} / \mathrm{H}]$ and $[\mathrm{Mg} / \mathrm{Fe}]$ distributions of the subsamples are very similar to those of the total samples inside each disc, suggesting that the $r_{\text {per }}$ peaks/bumps are not due to contamination from the thin/thick discs.

and call the stars composing the high $r_{\text {per }}$ peak the migrators, and the ones composing the low $r_{\text {per }}$ peak the non-migrators. In the GMM, 55 per cent of the thin disc stars are classified as migrators, while this fraction decreases to 38 per cent for thick disc stars - see Fig. 3 .

Finally, the right-hand column shows that the thin and thick discs have similar $z_{\max }$ distributions, peaking at $0.5 \lesssim z_{\max } / \mathrm{kpc} \lesssim 1$. Importantly, there is a large fraction of chemical thick disc stars at small $z_{\max }$, i.e. the region associated with the geometric thin disc. This large fraction is partially due to the adopted geometric cut $(d<2 \mathrm{kpc})$, which naturally suppresses the high $z_{\max }$ portion of the thick disc. However, in Appendix B we show that even selecting stars with $|z|<6 \mathrm{kpc}$, the fraction of chemical thick disc stars with low $z_{\max }$ is still significant. Finally, it is interesting to note that, for both the thin and thick discs, the migrator and non-migrator subsamples have similar $z_{\max }$ distributions as the total samples.

\section{$4 N$-BODY + SPH SIMULATION OF A CLUMPY GA LA XY}

We now explore an $N$-body + smooth particle hydrodynamics (SPH) simulation of the formation of an isolated galaxy that forms clumps in its early evolution. In these clumps, the star formation rate density is high and Type II supernovae rapidly enrich the surrounding medium with $\alpha$-elements. The clumps scatter $\alpha$-rich stars to high $|z|$ ( $\alpha$-poor stars are also scattered, but they are born further the clumps and therefore are less likely to be strongly scattered), giving rise to a geometric thick disc (as in the mechanism proposed by Bournaud et al. 2009). After $\approx 4 \mathrm{Gyr}$, the clumps stop forming because of the declining gas mass fraction and any remaining clumps are either disrupted or sink to the centre; thus clumps only affect the galaxy's early evolution. This scenario was shown to produce a chemical bimodality (Clarke et al. 2019), density profiles (Beraldo e Silva et al. 2020), and a Splash population (Amarante et al. 2020b) very similar to those observed in the MW (Mackereth et al. 2017; Holtzman et al.

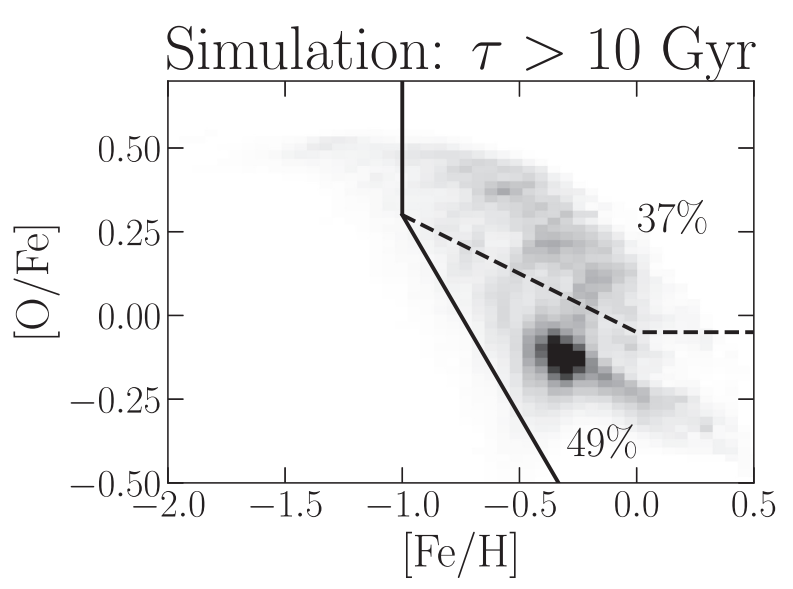

Figure 4. 2D histogram in chemical space for old stars in the simulation. Like in the MW (Fig. 2), a chemical bi-modality is already present in the very first Gyr of evolution. Dashed lines define the chemical thick and thin discs.

2018; Belokurov et al. 2020). Finally, co-formation of the thin and thick discs is a natural prediction of this scenario.

In this section, we first show that the clump scenario reproduces the observational trends discussed above. Then, we analyse the simulation's time evolution to unravel the secular evolution driving the MW to exhibit those trends.

\subsection{Simulation details}

For the initial conditions, we use a five times higher mass resolution version of that described in Clarke et al. (2019). A gas corona with $5 \times 10^{6}$ particles in hydrostatic equilibrium is placed within an NFW (Navarro, Frenk \& White 1997) dark matter halo which constitutes 90 per cent of the total mass. The virial radius and mass of the dark matter halo are $r_{200} \simeq 200 \mathrm{kpc}$ and $M_{200}=10^{12} \mathrm{M}_{\odot}$, respectively, 
and it also has $5 \times 10^{6}$ particles. While the gas corona has the same radial density profile, it constitutes only 10 per cent of the mass. No additional baryons or stars are present at $t=0$. The spin parameter of the gas corona is set to $\lambda=0.065$ (Bullock et al. 2001). The gas corona and the dark matter halo have softening lengths of $\epsilon=50$ and $100 \mathrm{pc}$, respectively. Star particles forming from the gas also have a softening of $\epsilon=50 \mathrm{pc}$.

These initial conditions are evolved for $13 \mathrm{Gyr}$ with GASOLINE (Wadsley, Stadel \& Quinn 2004; Wadsley, Keller \& Quinn 2017) with sub-grid models for the star formation, feedback and gas cooling, including the metal-line cooling of Shen, Wadsley \& Stinson (2010). The gas cools and settles into a disc. Once its density exceeds a certain threshold (set to $0.1 \mathrm{~cm}^{-3}$ ), star formation starts (with an efficiency set to 5 per cent) from gas particles with temperature below $15000 \mathrm{~K}$ which are part of a converging flow. We use the blast wave supernova feedback (Stinson et al. 2006), which couples, as thermal energy, 10 per cent of the $10^{51}$ erg per supernova to the interstellar medium. Gas mixing uses turbulent diffusion as described by Shen et al. (2010). A base time-step of $\Delta t=5 \mathrm{Myr}$ is used, with timesteps refined such that $\delta t=\Delta t / 2^{n}<\eta \sqrt{\epsilon / a_{g}}$, where we set the refinement parameter $\eta=0.175$. We set the opening angle (for the tree-code gravity calculation) to $\theta=0.7$. The time-steps of gas particles additionally satisfy the condition $\delta t_{\text {gas }}=\eta_{\text {courant }} h /[(1+\alpha) c$ $\left.+\beta \mu_{\max }\right]$, where $\eta_{\text {courant }}=0.4, h$ is the SPH smoothing length set over the 32 nearest-neighbour particles, $\alpha$ and $\beta$ are the linear and quadratic viscosity coefficients, and $\mu_{\max }$ is described by Wadsley et al. (2004).

Oxygen and iron yields from SN II and SN Ia are taken from Raiteri, Villata \& Navarro (1996), and we also use Padova stellar lifetimes to determine SN II rates, while SN Ia rates are determined from those same lifetimes in a binary evolution model.

We use data from the last snapshot, at $13 \mathrm{Gyr}$, and select star particles older than $10 \mathrm{Gyr}$ at the Solar torus, i.e. within a torus defined by circles at $R=8 \pm 2 \mathrm{kpc}$, for an approximate match with the selection criteria used for the MW. For brevity, we will refer to this region as the Solar Neighbourhood in the simulation. We compute the total gravitational potential at this final snapshot and integrate each orbit for 10 dynamical times using AGAMA (Vasiliev 2019). Finally, we calculate pericentre and apocentre radii, as well as eccentricities, using equation (1), and $z_{\max }$.

\subsection{Simulation results and comparison to the MW}

Fig. 4 shows a 2D histogram of $[\mathrm{O} / \mathrm{Fe}]$ versus $[\mathrm{Fe} / \mathrm{H}]$ for old star particles $(\tau>10 \mathrm{Gyr})$ in the simulation. The immediate conclusion is that, as in the MW, the simulated galaxy develops the chemical bi-modality associated with the thick/thin discs in the very first Gyr of its evolution, indicating a rapid formation of the two components. The straight lines define chemical thick and thin discs (above and below the dashed lines, respectively), similar to those defined in the MW (Fig. 2,upper panel). The thick (thin) disc comprises 37 per cent (49 per cent) of the total. If we instead select star particles older than $11 \mathrm{Gyr}$, we have 49 per cent (34 per cent) for thick (thin) disc. These numbers do not match in detail those in the cross-match with APOGEE-DR16 (Fig. 2 and Table B1). However, precise comparisons would require detailed modelling of the APOGEEDR16 selection function, which is beyond the scope of this work.

Fig. 5 (upper panel) shows the distribution of stars in the $r_{\text {per }}$ versus $e$ plane of the simulation ( $N=531519$ star particles). As in the MW (Fig. 1), the data peak along a line of approximately constant $r_{\text {apo }}$, at $8 \lesssim r_{\text {apo }} / \mathrm{kpc} \lesssim 9$, and is mostly concentrated in the region $6 \lesssim$
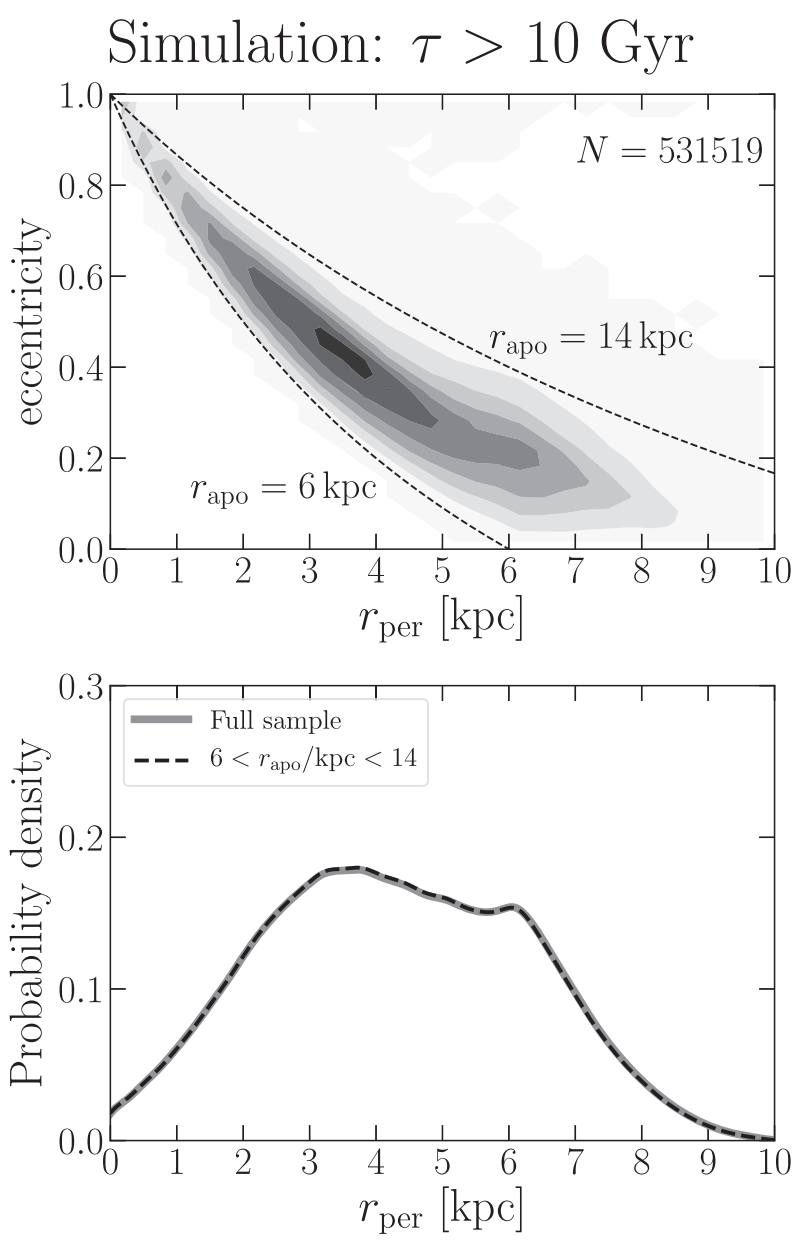

Figure 5. Top panel: distribution of old stars in eccentricity versus pericentric radius for the simulation Solar Neighbourhood. Bottom panel: KDEs of $r_{\text {per }}$ : for the full sample (grey) and for $6<r_{\text {per }} / \mathrm{kpc}<14$ (black dashed). As in the MW (Fig. 1), we identify two peaks, mainly (but not only) associated with the thick and thin disc, from the left- to right-hand side. The peak due to the accreted halo in the MW is not present, since the simulated galaxy evolves in isolation.

$r_{\text {apo }} / \mathrm{kpc} \lesssim 14$ (dashed lines), reflecting the same selection effects present in our MW sample, as described in Section 3.1. The bottom panel shows the $r_{\text {per }} \mathrm{KDE}$ for this sample (full grey) and after the restriction to the region $6 \lesssim r_{\text {apo }} / \mathrm{kpc} \lesssim 14$ (dashed black), which reduces the sample to $N=513907$ star particles. This restriction leaves the KDE essentially identical to the original one. We use this restriction in the rest of the analysis for the same reason as we did with the MW data, i.e. to avoid orbits with extreme or unrealistic kinematic properties, which can be introduced by the assumed potential.

The simulated galaxy lacks the first peak at $r_{\text {per }} \approx 0.5 \mathrm{kpc}$, which in the MW is associated with the accreted halo; this identification is strengthened by the fact that the simulated galaxy evolves in isolation, with no accretion events. The two other MW peaks are present in the simulation, at $r_{\text {per }} \approx 3.5$ and $6 \mathrm{kpc}$. The positions of these peaks differ from those in the MW (Fig. 1), as well as the eccentricity values at the peaks. However, these differences are not fundamentally important for our conclusions, since we are interested in comparing the trends, while the simulated galaxy is not intended to be an exact replica of the MW. Next, we show that indeed these two peaks are mostly, but not only, composed of thick and thin disc stars, respectively. For a 

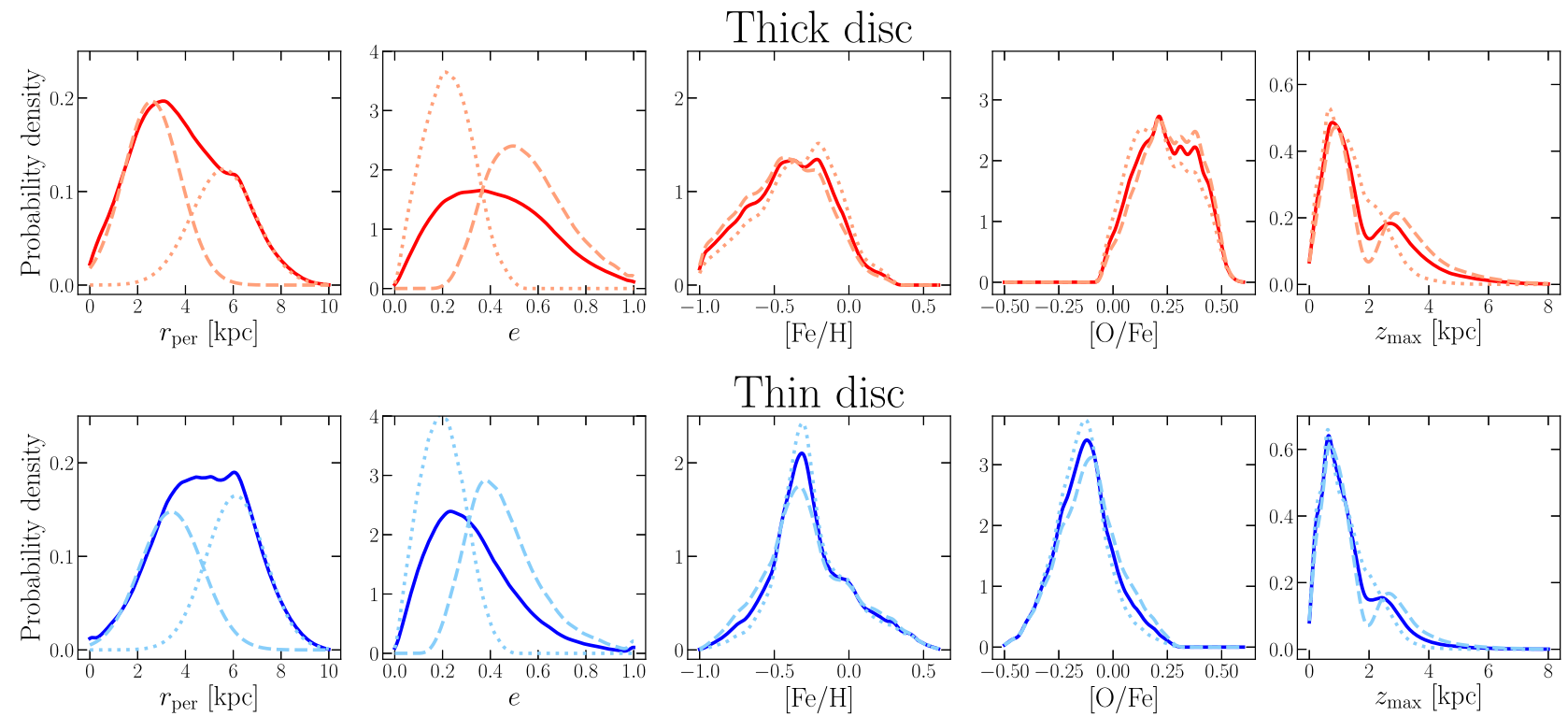

Figure 6. KDEs for kinematic quantities and chemical abundances for the chemical thin and thick discs in the simulation. As in the MW (Fig. 3), both discs have two peaks/bumps in the $r_{\text {per }}$ distribution, split into the two Gaussians (dotted and dashed curves in the first column). In other columns, dotted and dashed curves show the KDEs of the respective quantities for these two subsamples. The larger $r_{\text {per }}$ subsample (dotted) is characterized by lower eccentricities while the lower $r_{\text {per }}$ subsample (dashed) is characterized by higher eccentricities. As in the MW, the chemical abundance distributions of these subsamples are very similar to that of the total sample for each disc, suggesting that the $r_{\text {per }}$ peaks/bumps are not due to contamination from the thin/thick discs. The relative amplitudes of the two $r_{\text {per }}$ peaks/bumps in the thin and thick discs are similar those observed in the MW - see Fig. 3.

better comparison with the MW, in what follows we exclude the most metal-poor star-particles (those to the left of the solid line in Fig. 4).

Fig. 6 shows the KDE of the kinematic quantities and chemical abundances obtained in the simulation, for the thick disc (upper panels) and thin disc (bottom panels), to be compared to Fig. 3. The $r_{\text {per }}$ distributions of both thin and thick discs are bi-modal: The thick disc peaks at $r_{\text {per }} \approx 3 \mathrm{kpc}$ and has a bump at $r_{\text {per }} \approx 6 \mathrm{kpc}$, while the thin disc has a peak at $r_{\text {per }} \approx 4 \mathrm{kpc}$ and another, of slightly larger amplitude, at $r_{\text {per }} \approx 6 \mathrm{kpc}$. The relative amplitudes of these peaks and bumps agree well with those seen in the MW (Fig. 3).

We repeat the procedure used for the MW and use a GMM to split the $r_{\text {per }}$ distributions of the thin and thick discs into two Gaussians each (dashed and dotted curves in the left-hand panels). Then, for subsamples defined according to the highest probability of belonging to one or the other Gaussian component, we calculate the KDEs of other quantities, shown in the respective panels of Fig. 6. The eccentricities in the thin disc peak at $e \approx 0.2$ and have a narrower distribution than the thick disc, which peaks at $e \approx 0.35$. However, the thin disc has a tail for large eccentricities and both distributions are broader than those in the MW (Fig. 3, second column).

The $[\mathrm{Fe} / \mathrm{H}]$ and $[\mathrm{O} / \mathrm{Fe}] \mathrm{KDEs}$ are shown in the third and fourth columns. The subsamples (dotted and dashed lines) have KDEs very similar to the total samples, indicating that, as in the MW, the peaks and bumps in the $r_{\text {per }}$ distribution are not due to contamination between the thin and thick discs. Finally, the right-hand column shows the $z_{\max }$ distribution. The thick disc distribution is broader than that of the thin disc. It is interesting to note that the simulation $z_{\max } \mathrm{KDE}$ is bi-modal, while this bi-modality is apparently not present in Fig. 3. However, this bi-modality, associated with discrete tracks in a plot of $z_{\max }$ versus $r_{\text {apo }}$, has been observed in larger samples in the MW (Haywood et al. 2018), and shown to result from different orbital families in the adopted axisymmetric potential model - see Amarante, Smith \& Boeche (2020a) and Koppelman, Hagen \& Helmi (2020).
Thus, we conclude that the simulation reproduces the features observed for the old stars in the MW, mainly

(i) a chemical bi-modality comprising the thin and thick discs is already present in the very first Gyr of evolution, with relative contributions comparable to those in the MW;

(ii) the $r_{\text {per }}$ distribution has two peaks that can be mainly attributed to the thin and thick discs, although both peaks have contributions from both discs and this is not due to contamination;

(iii) $\sim 1 / 2$ of the old thin disc stars are associated to the high $r_{\text {per }}$ peak, while that fraction is $\sim 1 / 3$ for the thick disc;

(iv) stars that mostly contribute to the high $r_{\text {per }}$ peak are those with smaller eccentricities; and, finally,

(v) a significant fraction of chemical thick disc stars have low $z_{\max }$, i.e. overlap with the geometric thin disc.

We now analyse the simulation evolution to show that stars at the high $r_{\text {per }}$ peak formed at smaller radii and moved outwards due to radial migration, suggesting that the same happened in the MW.

\subsection{Time evolution and radial migration}

\subsubsection{Migrators all over the disc}

We start this section by comparing the current position (at $t=13 \mathrm{Gyr}$ ) of old star particles on nearly-circular orbits with the position they had at $t=5 \mathrm{Gyr}$. This earliest snapshot is chosen right after the early turbulent period when the clumps are still present, since we are now interested in the subsequent secular evolution. We select star particles older than $10 \mathrm{Gyr}$, but without any geometric cut. For each particle we calculate the circularity $\lambda_{\mathrm{c}}=L_{z} / L_{\mathrm{c}}(E)$, where $L_{z}$ is the angular momentum $z$-component and $L_{\mathrm{c}}(E)$ is the angular momentum of a circular orbit at the same energy $E$. Then, we select particles on nearly-circular orbits $\left(\lambda_{c}>0.9\right)$ and split this sample into chemical thin and thick discs (as defined in Fig. 4). 


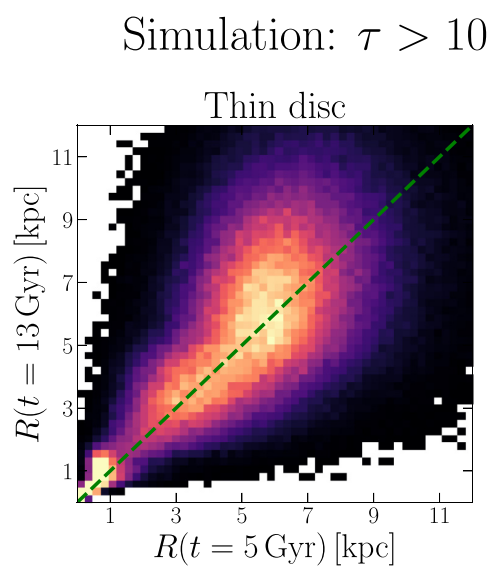

$\operatorname{Gyr}\left[L_{z} / L_{c}(E)>0.9\right]$
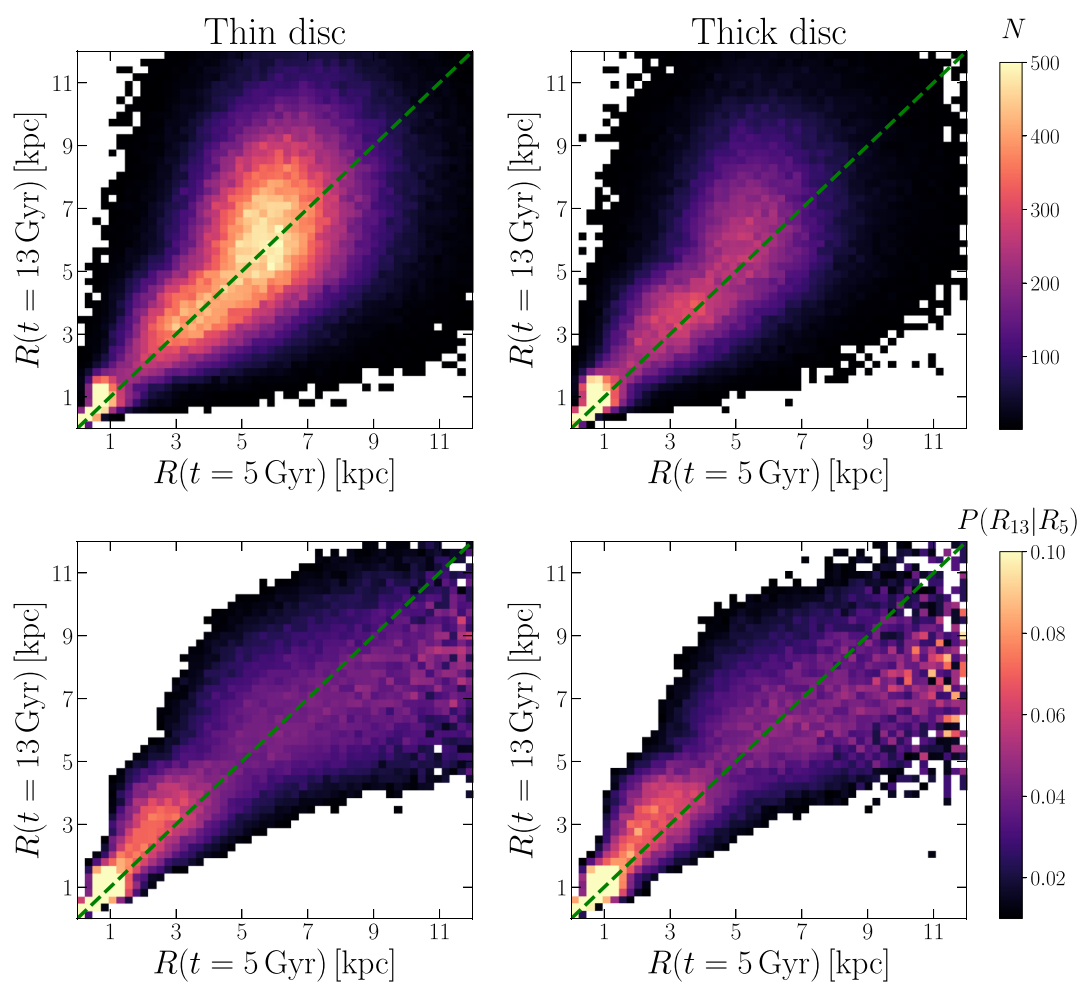

Figure 7. $2 \mathrm{D}$ histograms of (cylindrical) radii $R(t=13 \mathrm{Gyr})$ versus $R(t=5 \mathrm{Gyr})$ for old star particles on nearly-circular orbits, split into thin/thick discs (left/right-hand panels). Top panels are colour-coded by total numbers, while bottom panels are column-normalized. The large spread around the diagonal shows the effect of radial migration (churning) produced by spiral arms. This affects the thin and thick discs similarly, although particles on nearly-circular orbits (those most affected) are less numerous in the thick disc.

Fig. 7 shows 2D histograms of the cylindrical radius at different times, $R(t=13 \mathrm{Gyr})$ versus $R(t=5 \mathrm{Gyr})$ for this sample, split into thin (left) and thick (right) discs. Colours in the top row represent total numbers, while the bottom row histograms are column-normalized. If particles kept on orbits at the same early radii they had at early times, they would concentrate along the diagonal dashed line. However, a large spread is observed, with many particles changing radii by several kpc. Since we select particles on nearly-circular orbits, we suppress the possibility of migration by blurring, i.e. by oscillations around the guiding radii. Thus, Fig. 7 shows the effect of radial migration (churning) all over the disc, i.e. particles moving from nearly-circular orbits to other nearly-circular orbits, as proposed by Sellwood \& Binney (2002). Importantly, although the number of nearly-circular orbits in the thick disc is smaller than in the thin disc, as shown in the top panels, those orbits are similarly affected by radial migration in the two discs, as shown in the bottom panels.

For a compact representation of the radial migration effect, we calculate $\Delta R \equiv R(13 \mathrm{Gyr})-R(5 \mathrm{Gyr})$ for star particles on nearlycircular orbits $\left(\lambda_{\mathrm{c}}>0.9\right)$ and show its KDE in Fig. 8. The blue (red) curve represents the thin (thick) disc. The two discs are similarly affected by radial migration: Both KDEs have a narrow peak near $\Delta R=0$, and are skewed, with a longer tail to the right, signing a net movement outwards, a consequence of the density decrease outwards. We find $\left\langle(\Delta R)^{2}\right\rangle^{1 / 2} \sim 2.3 \mathrm{kpc}$ for the thin disc (similar to that found by Roškar et al. 2008) and $\sim 2.1 \mathrm{kpc}$ for the thick disc. Frankel et al. (2020) found $\left\langle(\Delta R)^{2}\right\rangle^{1 / 2} \sim 2.6 \mathrm{kpc} \sqrt{\tau / 6 \mathrm{Gyr}}$ for MW thin disc stars spanning $5 \lesssim R / \mathrm{kpc} \lesssim 13$. For $\tau=8$ Gyr (time between the two snapshots), this results in $\left\langle(\Delta R)^{2}\right\rangle^{1 / 2} \sim 3 \mathrm{kpc}$, while applying the same geometric cut, we find $\left\langle(\Delta R)^{2}\right\rangle^{1 / 2} \sim 2.6 \mathrm{kpc}$ for the thin disc. Therefore, the extent of migration in the simulation is comparable to that in the MW.

\subsubsection{Migrators in the Solar Neighbourhood}

Having shown the effect of radial migration all over the thin and thick discs, we now investigate its role in producing the high $r_{\text {per }}$ peak shown in Figs 1, 3, 5, and 6. We go back to the original geometric cut and select, at $t=13 \mathrm{Gyr}$, star particles older than $10 \mathrm{Gyr}$ in the simulation Solar Neighbourhood. Then, we track these same particles at different snapshots from 5 to $13 \mathrm{Gyr}$. At each snapshot, we integrate the particles orbits in the respective potentials and determine the $r_{\text {per }}$, $r_{\text {apo }}$ and eccentricity for each particle.

Fig. 9 (two left-hand columns) shows the $r_{\text {per }}$ KDEs (solid lines) for the thin (blue) and thick disc (red) at different snapshots (rows). Similarly to Figs 3 and 6, at the final snapshot (bottom panels), we split our $r_{\text {per }}$ sample into two Gaussian components using a GMM (dashed and dotted curves in the bottom panels) and use these components to define two subsamples. Then, for these fixed subsamples we calculate, at each snapshot, the $r_{\text {per }}$ KDEs, shown as dotted and dashed curves in the remaining plots (weighted according to the Gaussian weights obtained at the final snapshot).

At $5 \mathrm{Gyr}$, the full sample distributions (thick solid lines) are unimodal for both thin and thick discs, peaking at $r_{\text {per }} \approx 4 \mathrm{kpc}$ for the thin disc and at $r_{\mathrm{per}} \approx 3 \mathrm{kpc}$ for the thick disc, which must reflect 


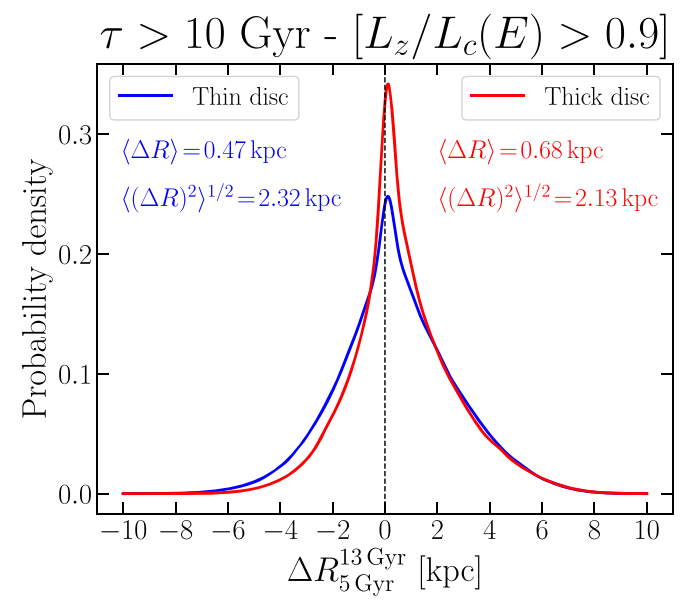

Figure 8. KDEs of $\Delta R \equiv R(t=13 \mathrm{Gyr})-R(t=5 \mathrm{Gyr})$ for the thin (blue) and thick (red) discs. Both KDEs peak near $\Delta R=0$, but have long tails, with star particles migrating by several kpc. The positive skewness indicate a net outward movement. differences in average formation radii for these two components. From the top to bottom panels, we see that while the positions of these peaks are approximately conserved, the distributions get progressively positively skewed, until another peak appears at $r_{\text {per }} \approx 6 \mathrm{kpc}$ for the thin disc and a bump at this same location for the thick disc.

For both thin and thick discs, while the low $r_{\text {per }}$ Gaussian component (dashed) stays approximately fixed at the same position for different snapshots, the high $r_{\text {per }}$ component (dotted) moves to the right and becomes skewed to large values, producing the high $r_{\text {per }}$ peak at the final snapshot. This shows that the high $r_{\text {per }}$ peak is characterized by stars which, on average, formed in inner radii and move outwards. The hashed regions in Fig. 9 are defined as those star-particles which, at the final time, have $r_{\text {per }}>7 \mathrm{kpc}\left(r_{\text {per }}>6 \mathrm{kpc}\right)$ for the thin (thick) disc. Beyond these values, the contribution from the low $r_{\text {per }}$ peak Gaussian component is negligible, allowing to show the effect of migration in a cleaner way. Some star-particles move at least $4 \mathrm{kpc}$ outwards in $2 \mathrm{Gyr}$ (from 11 to $13 \mathrm{Gyr}$ ).

Besides the tendency of a net outward movement observed in Section 4.3.1, this is a consequence of the inside-out growth of the galactic disc and of selecting old stars in the Solar Neighbourhood,
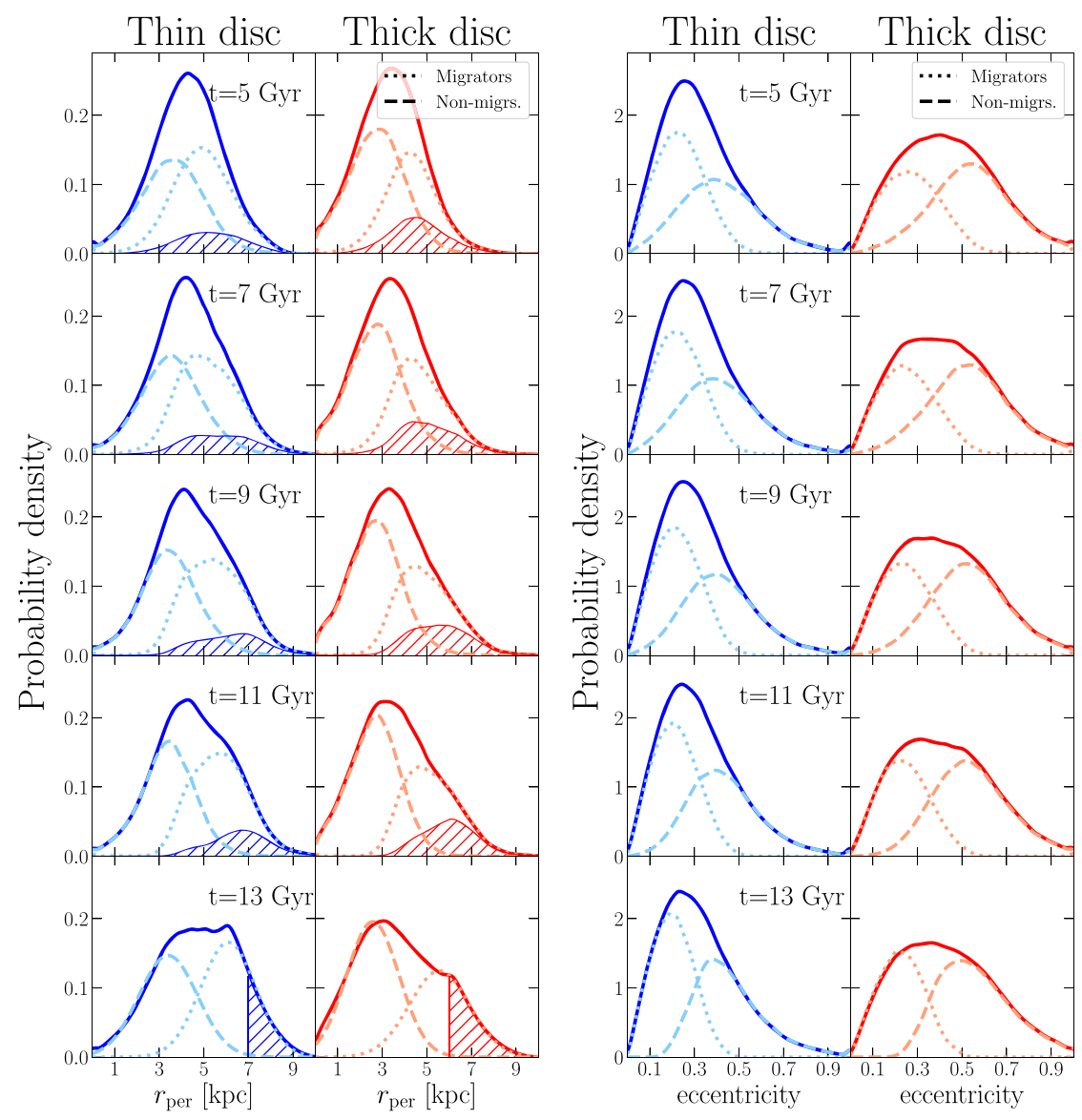

Figure 9. Left-hand panel: time evolution of the $r_{\text {per }}$ KDE in the thin (blue) and thick (red) discs of the simulation. Old star particles ( $\tau>10$ Gyr) are selected and split into two Gaussians at $t=13 \mathrm{Gyr}$. These fixed samples and subsamples are analysed at earlier times (same particles considered in all snapshots). Right-hand panel: time evolution of the eccentricities KDE in the thin and thick discs for the fixed samples defined in the left-hand panels. In both thin and thick discs, the low $r_{\text {per }}$ subsample (dashed) has higher eccentricities, and thus is less affected by radial migration, staying nearly fixed in place (the non-migrators). The high $r_{\text {per }}$ subsample (dotted) is that on nearly-circular orbits, and thus is significantly affected by radial migration and tends to move outwards (the migrators), developing a positive skewness. At $t=13 \mathrm{Gyr}$, the migrators produce the high $r_{\text {per }}$ peaks. Hatched areas show samples selected at $t=13 \mathrm{Gyr}$ as those with $r_{\text {per }}>7 \mathrm{kpc}\left(r_{\text {per }}>6 \mathrm{kpc}\right)$ for the thin (thick) disc, showing the effect in a sample less contaminated by non-migrators. The migrators' eccentricity distributions are conserved, agreeing with expectations for radial migration. Non-migrators exhibit some heating, with nearly-circular orbits at $t=5 \mathrm{Gyr}$ getting more eccentric with time. 
which was poorly populated at early stages. Stars can visit the Solar Neighbourhood by oscillating around their guiding radius (blurring, most relevant for eccentric orbits) or via radial migration (churning). As mentioned in Section 3.3, radial migration is more effective for low-eccentricity orbits and it does not significantly change these eccentricities. In other words, radial migration (churning) typically moves stars from circular orbits to other circular orbits (Sellwood \& Binney 2002; Roškar et al. 2012). In Fig. 6, we showed that the high $r_{\text {per }}$ component is the one with the lower eccentricities, in agreement with the MW (Fig. 3). Moreover, Fig. 9 (two right-hand columns) shows the KDEs of the eccentricity at the same times and for the same samples as in the left-hand panels. The eccentricities for those stars which will produce the high $r_{\text {per }}$ peak in the left-hand panels (dotted lines) do not show a significant evolution. On the other hand, the other subsample, which will produce the low $r_{\text {per }}$ peak in the two left-hand columns (dashed lines), shows some evolution, moving the left-hand tail to the right, i.e. with any low-eccentricity orbits gradually becoming more eccentric (heating).

Therefore, these results strongly suggest that the process responsible for moving a population outwards and producing the high $r_{\text {per }}$ peak (in both the simulation and in the MW) is radial migration (churning), which justifies calling this population 'the migrators'.

\section{DISCUSSION}

\subsection{Implications for the disc formation}

As mentioned in the Introduction, some theoretical models predict a sequential formation of thin and thick discs, with the thin disc becoming a significant component only after the thick disc has formed. In such scenarios, a significant population of old thin disc stars is unexpected. In Section 3, we showed several lines of evidence for such a population in the MW (Figs 2 and 3), with thin (thick) disc stars comprising 29 per cent (54 per cent) of the sample.

The existence of a significant old thin disc population suggests that the thin disc starts forming earlier than generally assumed, having a large formation time overlap with the thick disc, and this is the main result of this work. Therefore, models that predict a sequential formation of the thin/thick discs, such as the upsidedown scenario, are disfavored by our results. Also disfavoring this scenario is our finding of a large fraction of chemical thick disc stars with low $z_{\max }$ (peaking at $z_{\max } \approx 1 \mathrm{kpc}-$ see Fig. B1), which suggests two possible explanations: Either, in line with the upsidedown scenario, these low $z_{\max }$ stars were born at high $|z|$ and settled to the geometric thin disc, or the chemical thick disc stars were born at low $|z|$ and a fraction of these stars were scattered to high $|z|$, leaving a significant fraction at low $z_{\max }$. Since stars constitute a collisionless component, one expects them to get kinematically hotter under secular evolution (unless some fine-tuned mechanism is advocated to kinematically cool these stars), thus making the second possibility more plausible. Recently, Ciucă et al. (2020) estimated ages from APOGEE-DR14 survey data using a Bayesian machine learning framework. Their results indicate that, while the inner and outer discs seem to have different chemical evolutionary pathways, there is a smooth transition between the formation of the thick disc and the inner thin disc (see also Feuillet et al. 2019). A significant time-overlap in the formation of these components is also apparent in their results (although these authors did not explore this topic further), which are thus complementary to the results found by us.

On the other hand, some models do predict an early co-formation of thin and thick discs and thus the existence of a conspicuous old thin disc population. In the accretion model, Abadi et al. (2003) predict that 15 per cent of the current thin disc stars should be older than 10 Gyr. In this picture an old thin disc is built up from several disrupted satellites, each with unique chemical signatures, geometry, and kinematics. Thus, Abadi et al. (2003) predicted that this old thin disc should show distinct chemo-kinematic correlations. In our sample, the subsamples of stars classified as migrators and nonmigrators (based on $r_{\text {per }}$, which is similar to splitting by eccentricity) have chemical abundance distributions very similar to the total samples (Fig. 3). This suggests that the correlations predicted in the accretion scenario are not observed in the MW.

In the major merger scenario (Quinn et al. 1993; Villalobos \& Helmi 2008; Helmi et al. 2018), the thick disc is produced by a merger whose progenitor is massive enough to vertically heat a protodisc, and an old thin disc remnant is expected. Villalobos \& Helmi (2008) predict that the mass fraction of this remnant thin disc is 15-25 per cent of the total stellar mass at the end of the merger. Note that this refers to a geometric definition, while the chemical thin disc is generally seen as forming only after the merger (see e.g. Grand et al. 2020), in which case the significant old chemical thin disc population found in this work is unexpected. On the other hand, if a chemical thin disc population were present in the proto-disc, a significant fraction of these stars should have been vertically heated and should have large $z_{\max }$, similar to thick disc stars. However, the thin disc $z_{\max }$ distribution does not extend as much as the thick disc to large values (see Fig. B1, dashed lines). Thus, also the major merger scenario seems disfavored by our results. Additionally, possible concerns with this scenario have been recently raised on the basis of asteroseismic age estimates of thick disc stars. Montalbán et al. (2020) estimated ages (within $\sim 10$ per cent) of metal-poor stars $([\mathrm{Fe} / \mathrm{H}]<-0.5)$, finding that part (if not all) of thick disc stars are as old as (or even slightly older than) stars accreted in the last major merger, thus concluding that this event was not the main trigger to the thick disc formation.

Whatever the case may be, these discussions suggest that the thick disc formation is still puzzling, which should motivate further studies of alternative scenarios. A compelling possibility is offered by the clumpy scenario explored in our simulation (Section 4), in which the early galaxy forms clumps and there are two main star formation channels: $\alpha$-rich stars are born in clumps and tend to be scattered to high $|z|$ and reasonably large eccentricities (forming the thick disc), while the formation of $\alpha$-poor stars is more broadly distributed over the disc. This scenario has been shown to generate a chemical bi-modality, geometric thin+thick discs and a Splash population in good agreement with those observed in the MW (Clarke et al. 2019; Amarante et al. 2020b; Beraldo e Silva et al. 2020). Most importantly, an early start for the thin disc formation, with a large time overlap with the thick disc formation, is a direct prediction of this scenario - see fig. 11 in Clarke et al. (2019). Additionally, this scenario also naturally predicts that a fraction of the chemical thick disc should overlap with the geometric thin disc, i.e. should have low $z_{\max }$, as found in this work for the MW - see Fig. B1.

\subsection{Radial migration: thin disc versus thick disc}

The analysis of Section 4.3 showed evidence for the role of radial migration in the simulation, changing the guiding radii of stars in nearly-circular orbits typically by $\left\langle(\Delta R)^{2}\right\rangle^{1 / 2} \sim 2 \mathrm{kpc}$ in a few Gyr. We showed that a consequence of this is the appearance of a peak in the $r_{\text {per }}$ distribution of old stars, with properties very similar to those found in the MW. Interestingly, this evidence is present in both the chemical thin and thick discs - see Fig. 9. 
Going back to Fig. 6, we can now evaluate differences in the relevance of radial migration for the thin and thick discs. As already mentioned, migrators (non-migrators) are characterized by low (high) eccentricities. On the other hand, inside the thin or thick discs, the $z_{\max }$ distribution in our simulation (Fig. 6, right-hand panels) is similar for migrators and non-migrators (dashed and dotted curves). This shows that whether a star will be affected by radial migration depends essentially on its eccentricity, but not much on its vertical excursion, in agreement with Solway, Sellwood \& Schönrich (2012). In other words, the extent to which thin and thick discs are differently affected by radial migration is determined by their eccentricity distributions, and not by their different scale heights: the thin disc is more affected by radial migration because a large fraction of its orbits have low eccentricities, while that fraction is lower in the thick disc and it is in this sense that the thick disc is less affected by radial migration. In fact, for particles in nearly-circular orbits all over the disc, thin and thick discs are equally affected by radial migration - see Fig. 8. Moreover, selecting particles at the Solar Neighbourhood, and judging by the similarity of the $r_{\text {per }}$ KDEs of thin and thick discs at $5 \mathrm{Gyr}$ (Fig. 9) and the similar positions of the high $r_{\text {per }}$ peak at $13 \mathrm{Gyr}$, one can say that the migrators in both the thin and thick discs are equally influenced by radial migration, i.e. move by similar amounts in the same time interval.

Recently, Mikkola, McMillan \& Hobbs (2020) also concluded that the vertical excursions are not of much relevance to radial migration in simulated galaxies if the disc is more dominant with respect to the dark matter halo, while vertical excursions can be more relevant for galaxies with more dominant haloes. Thus, the disc and halo masses relative contributions can have some importance on our results regarding the simulated galaxy and the MW. Interestingly, Cole \& Binney (2017) find that baryons dominate the MW's gravitational potential in the disc out to about the Solar Neighbourhood, which, considering the conclusions of Mikkola et al. (2020), reinforces our finding of a significant migration in the thick disc of the MW.

\subsection{The conundrum of RR Lyrae in the thin disc}

As discussed in the Introduction, in sequential formation scenarios, where the thin disc only forms after the thick disc has formed, RR Lyrae (which have ages $\gtrsim 10 \mathrm{Gyr}$ ) are not expected to be found in the thin disc. The recent detection of a population of thin disc RR Lyrae then appears as a conundrum in such scenarios (Prudil et al. 2020). One solution of this conundrum was proposed by Iorio \& Belokurov (2021). Using a sample of $~ 70000$ RR Lyrae in GaiaDR2, they confirmed a subpopulation with high rotational velocity and low $|z|$, typical of the thin disc. They found that their velocity dispersion is almost a factor of 2 smaller than that of a sample with age $\tau=10 \mathrm{Gyr}$; comparing with the empirically determined agevelocity dispersion relation of Sharma et al. (2020), they concluded that this population has velocity dispersion similar to that of a 2-Gyr-old population. They suggested that these might be some other population of stars that mimics RR Lyrae. We note, however, that a rotationally selected sample is by definition kinematically biased (towards small velocity dispersions), while the sample used by Sharma et al. (2020) to fit the AVR is complete, and this could potentially lead to the conclusion of a younger population.

The solution provided by our results is that a thin disc population of old RR Lyrae is not a problem in the first place. Our results show that the existence of these old thin disc stars suggests an early co-formation of thin and thick discs, a scenario in which thin disc RR Lyrae stars are naturally accommodated. In this sense, the
RR Lyrae are no different from other old populations, a fraction of which is also $\alpha$-poor, thin, and rapidly rotating like the thin disc.

\section{CONCLUSIONS}

In this work, we select, from the Sanders \& Das (2018) catalogue, old stars $(\tau>10 \mathrm{Gyr})$ within $2 \mathrm{kpc}$ from the Sun. After several quality cuts, our sample consists of $N=23795$ (turnoffs + giants) stars with 6D phase-space coordinates from Gaia-DR2. We use these to integrate orbits in an assumed Galactic potential, determining peri- and apocentre radii, eccentricities and maximum vertical excursions. For a subsample of $N=1049$ (turnoffs + giants) stars, we obtain $[\mathrm{Mg} / \mathrm{Fe}]$ and $[\mathrm{Fe} / \mathrm{H}]$ abundances from APOGEE-DR 16 and chemically split it into halo, thin, and thick disc stars. The restricted size of this sample is associated with fundamental limitations of the observational data currently available, given the quality cuts required to simultaneously obtain good age estimates, 6D phasespace information, and chemical abundances. We compare the results with those from a simulated galaxy which forms clumps in its early evolution. Our conclusions are summarized as follows:

(i) Chemical thin disc stars represent a significant fraction of our sample of old stars, with star counts comparable to those of the chemical thick disc - see Table B1. We interpret this as evidence that the thin disc formation starts early, with a large time overlap with the formation of the thick disc.

(ii) The old stars $r_{\mathrm{per}}$ distribution has three peaks, one at $r_{\mathrm{per}} \approx 0.59 \mathrm{kpc}$, one at $r_{\mathrm{per}} \approx 5.4 \mathrm{kpc}$, and another at $r_{\mathrm{per}} \approx 7.14 \mathrm{kpc}$. This confirms the findings of Prudil et al. (2020), who showed evidence for three $r_{\text {per }}$ peaks with a sample of 314 RR Lyrae stars.

(iii) The first peak is associated with the stellar halo. The other two peaks have contributions from both the thin and thick discs.

(iv) In both the thin and thick discs, stars contributing to the second $r_{\text {per }}$ peak are those with higher eccentricities, while stars contributing to the third $r_{\text {per }}$ peak are those on nearly-circular orbits. We suggest (and confirm with the simulation) that the third peak is produced by stars which migrated from inner radii, while the second peak is mostly due to non-migrating stars.

(v) In the Solar Neighbouhood, $\sim 1 / 2(\sim 1 / 3)$ of the old thin (thick) disc stars can be classified as migrators.

(vi) Thin and thick discs are differently affected by radial migration inasmuch as they have different eccentricity distributions. Therefore, thick disc stars on nearly-circular orbits are as affected by radial migration as thin disc stars in nearly-circular orbits.

(vii) Our results disfavour sequential formation models, such as the upside-down scenario, where the thin disc only starts to be significant after the thick disc is formed.

(viii) Among the models that predict an early co-formation of thin and thick discs, the clumpy star formation scenario seems to be favoured by our results, which is demonstrated by a good agreement between observational and simulated trends.

\section{ACKNOWLEDGEMENTS}

We thank Eugene Vasiliev for help with the AGAMA library, Giuliano Iorio and Vasily Belokurov for discussions on their analysis of RR-Lyrae stars, and Stuart Anderson for highlighting typographic corrections. VPD and LBS are supported by STFC Consolidated grant \# ST/R000786/1. The simulations in this paper were run at the DiRAC Shared Memory Processing system at the University of Cambridge, operated by the COSMOS Project at the Department of Applied Mathematics and Theoretical Physics on behalf of the STFC 
DiRAC HPC Facility (www.dirac.ac.uk). This equipment was funded by BIS National E-infrastructure capital grant ST/J005673/1, STFC capital grant ST/H008586/1, and STFC DiRAC Operations grant ST/K00333X/1. DiRAC is part of the National E-Infrastructure. JA acknowledges The World Academy of Sciences and the Chinese Academy of Sciences for the CAS-TWAS fellowship.

\section{DATA AVAILABILITY}

The Sanders \& Das data set is publicly available from this URL. APOGEE-DR16 stellar abundances are available at this URL. The simulation data set used here is proprietary but can be shared for limited use on request to VPD (vpdebattista@gmail.com).

\section{REFERENCES}

Abadi M. G., Navarro J. F., Steinmetz M., Eke V. R., 2003, ApJ, 597, 21 Adibekyan V. Z., Santos N. C., Sousa S. G., Israelian G., 2011, A\&A, 535, L11

Agertz O. et al., 2020, preprint (arXiv:2006.06008)

Ahumada R. et al., 2020, ApJS, 249, 3

Amarante J. A. S., Smith M. C., Boeche C., 2020a, MNRAS, 492, 3816

Amarante J. A. S., Beraldo e Silva L., Debattista V. P., Smith M. C., 2020b, ApJ, 891, L30

Anders F. et al., 2014, A\&A, 564, A115

Belokurov V., Erkal D., Evans N. W., Koposov S. E., Deason A. J., 2018, MNRAS, 478, 611

Belokurov V., Sanders J. L., Fattahi A. et al., 2020, MNRAS, 494, 3880

Bensby T., Feltzing S., Oey M. S., 2014, A\&A, 562, A71

Beraldo e Silva L., Debattista V. P., Khachaturyants T., Nidever D., 2020, MNRAS, 492, 4716

Bird J. C., Kazantzidis S., Weinberg D. H., Guedes J., Callegari S., Mayer L., Madau P., 2013, ApJ, 773, 43

Bournaud F., Elmegreen B. G., Martig M., 2009, ApJ, 707, L1

Bovy J., Rix H.-W., Liu C., Hogg D. W., Beers T. C., Lee Y. S., 2012, ApJ, 753, 148

Bovy J., Rix H.-W., Schlafly E. F., Nidever D. L., Holtzman J. A., Shetrone M., Beers T. C., 2016, ApJ, 823, 30

Brook C. B., Kawata D., Gibson B. K., Freeman K. C., 2004, ApJ, 612, 894

Bullock J. S., Dekel A., Kolatt T. S., Kravtsov A. V., Klypin A. A., Porciani C., Primack J. R., 2001, ApJ, 555, 240

Chiappini C., Matteucci F., Romano D., 2001, ApJ, 554, 1044

Ciucă I., Kawata D., Miglio A., Davies G. R., Grand R. J. J., 2020, preprint (arXiv:2003.03316)

Clarke A. J. et al., 2019, MNRAS, 484, 3476

Cole D. R., Binney J., 2017, MNRAS, 465, 798

Feuillet D. K., Frankel N., Lind K., Frinchaboy P. M., García-Hernández D. A., Lane R. R., Nitschelm C., Roman-Lopes A., 2019, MNRAS, 489, 1742

Frankel N., Sanders J., Ting Y.-S., Rix H.-W., 2020, ApJ, 896, 15

Gilmore G., Reid N., 1983, MNRAS, 202, 1025

Glatt K. et al., 2008, AJ, 135, 1106

Grand R. J. J. et al., 2020, MNRAS, 497, 1603

Hall P., York M., 2001, Stat. Sin., 11, 515

Hayden M. R. et al., 2015, ApJ, 808, 132

Haywood M., Di Matteo P., Lehnert M. D., Snaith O., Khoperskov S., Gómez A., 2018, ApJ, 863, 113

Helmi A., Babusiaux C., Koppelman H. H., Massari D., Veljanoski J., Brown A. G. A., 2018, Nature, 563, 85

Holtzman J. A. et al., 2018, AJ, 156, 125

Iorio G., Belokurov V., 2021, MNRAS, in press

Ivezić Ž., Connelly A. J., VanderPlas J. T., Gray A., 2014, Statistics, Data Mining, and Machine Learning in Astronomy. Princeton Univ. Press

Jönsson H. et al., 2020, AJ, 160, 120

Kazantzidis S., Bullock J. S., Zentner A. R., Kravtsov A. V., Moustakas L. A., 2008, ApJ, 688, 254
Khoperskov S., Haywood M., Snaith O., Di Matteo P., Lehnert M., Vasiliev E., Naroenkov S., Berczik P., 2020, preprint (arXiv:2006.10195)

Koppelman H. H., Hagen J. H. J., Helmi A., 2020, preprint (arXiv:2009.04849)

Kroupa P., 2002, MNRAS, 330, 707

Lian J. et al., 2020, MNRAS, 497, 2371

Loebman S. R., Roškar R., Debattista V. P., Ivezić Ž., Quinn T. R., Wadsley J., 2011, ApJ, 737, 8

Loebman S. R., Debattista V. P., Nidever D. L., Hayden M. R., Holtzman J. A., Clarke A. J., Roškar R., Valluri M., 2016, ApJ, 818, L6

McMillan P. J., 2017, MNRAS, 465, 76

Mackereth J. T. et al., 2017, MNRAS, 471, 3057

Mackereth J. T. et al., 2019, MNRAS, 482, 3426

Meng X., Gnedin O. Y., 2020, preprint (arXiv:2006.10642)

Mikkola D., McMillan P. J., Hobbs D., 2020, MNRAS, 495, 3295

Montalbán J. et al., 2020, preprint (arXiv:2006.01783)

Muñoz-Mateos J. C., Gil de Paz A., Boissier S., Zamorano J., Jarrett T., Gallego J., Madore B. F., 2007, ApJ, 658, 1006

Navarro J. F., Frenk C. S., White S. D. M., 1997, ApJ, 490, 493

Nidever D. L. et al., 2014, ApJ, 796, 38

Pedregosa F. et al., 2011, J. Mach. Learn. Res., 12, 2825

Prudil Z., Dékány I., Grebel E. K., Kunder A., 2020, MNRAS, 492, 3408

Quinn P. J., Hernquist L., Fullagar D. P., 1993, ApJ, 403, 74

Raiteri C. M., Villata M., Navarro J. F., 1996, A\&A, 315, 105

Renaud F., Agertz O., Read J. I., Ryde N., Andersson E. P., Bensby T., Rey M. P., Feuillet D. K., 2020a, preprint (arXiv:2006.06011)

Renaud F., Agertz O., Andersson E. P., Read J. I., Ryde N., Bensby T., Rey M. P., Feuillet D. K., 2020b, preprint (arXiv:2006.06012)

Rojas-Arriagada A., Zoccali M., Schultheis M., Recio-Blanco A., Zasowski G., Minniti D., Jönsson H., Cohen R. E., 2019, A\&A, 626, A16

Roškar R., Debattista V. P., Quinn T. R., Wadsley J., 2012, MNRAS, 426, 2089

Roškar R., Debattista V. P., Stinson G. S., Quinn T. R., Kaufmann T., Wadsley J., 2008, ApJ, 675, L65

Sanders J. L., Das P., 2018, MNRAS, 481, 4093

Schönrich R., Binney J., 2009a, MNRAS, 396, 203

Schönrich R., Binney J., 2009b, MNRAS, 399, 1145

Sellwood J. A., Binney J. J., 2002, MNRAS, 336, 785

Sharma S. et al., 2020, preprint (arXiv:2004.06556)

Shen S., Wadsley J., Stinson G., 2010, MNRAS, 407, 1581

Silverman B. W., 1981, J. R. Stat. Soc, 43, 97

Solway M., Sellwood J. A., Schönrich R., 2012, MNRAS, 422, 1363

Stinson G., Seth A., Katz N., Wadsley J., Governato F., Quinn T., 2006, MNRAS, 373, 1074

VandenBerg D. A., Brogaard K., Leaman R., Casagrand e L., 2013, ApJ, 775, 134

Vasiliev E., 2019, MNRAS, 482, 1525

Vickers J. J., Smith M. C., 2018, ApJ, 860, 91

Villalobos Á., Helmi A., 2008, MNRAS, 391, 1806

Wadsley J. W., Stadel J., Quinn T., 2004, New Astron., 9, 137

Wadsley J. W., Keller B. W., Quinn T. R., 2017, MNRAS, 471, 2357

Yoshii Y., 1982, PASJ, 34, 365

\section{APPENDIX A: SILVERMAN TEST FOR NUMBER OF PEAKS}

In this section, we explain the test proposed by Silverman (1981) and used here to test for the number of peaks in the pericentre radius distribution of our sample. This test is based on the number of peaks produced by the data KDEs with varying window widths. A small window width produces a KDE with many peaks, possibly overfitting the data, while a large window width KDE produces a reduced number of peaks, possibly erasing important data structure. The null hypothesis $H_{0}$ is that the distribution has $k$ peaks against the hypothesis $H_{1}$ that it has $k+1$ or more peaks. The algorithm is as follows: For each number $k$ of peaks to be tested, starting from one, 
Table A1. Results for the test of statistical significance of $r_{\text {per }}$ peaks in Fig. 1.

\begin{tabular}{llll}
\hline$k$ & $h_{\text {crit }}$ & $P_{\text {Silverman }}$ & $P_{\mathrm{HY}}$ \\
\hline 1 & 0.632 & $\approx 1$. & $\approx 1$. \\
2 & 0.530 & $\approx 1$. & $\approx 1$. \\
3 & 0.207 & 0.523 & 0.662 \\
\hline
\end{tabular}

Notes. The first column $(k)$ is the number of peaks to be tested; the second column indicates the critical bandwidth, i.e. the minimum to produce at most $k$ peaks; the third column shows the probability for the number of peaks to be larger than $k$ in the original test of Silverman (1981); and the fourth column shows the probabilities after the correction/calibration of Hall \& York (2001). Models with $P>0.95$ can be excluded.

we define the critical window width $h_{\text {crit }}$ as the minimum width to produce at most $k$ peaks. We check the statistical significance of this $h_{\text {crit }}$ by bootstrap, re-sampling the data, and calculating a new $h_{\text {crit }}^{*}$ for each sample. At a given significance $\alpha$, we reject $H_{0}$ if, for an appropriate quantity $\lambda_{\alpha}$, the probability $P\left(h_{\text {crit }}^{*} / h_{\text {crit }}>\lambda_{\alpha}\right)>1-\alpha$. The main advantage of this method is that it does not rely on the Gaussianity assumption inherent in Bayesian information criterion (BIC) and Akaike information criterion (AIC) tests under a Gaussian Mixture Model - see Ivezić et al. (2014). Note that by 'peak' we mean a local maximum, as opposed to a 'bump' that is an interval where the curve is concave (seen from below) but not necessarily a maximum.

In Silverman (1981) original test, $\lambda_{\alpha}=1$, which is known to be conservative, i.e. less restrictive in rejecting models. For 1000 bootstrap samples, we obtain the probabilities shown in Table A1, third column. Despite the conservativeness of the test, models with $k=1$ or 2 peaks are rejected with high significance. Correcting and calibrating this test, Hall \& York (2001) obtained $\lambda_{\alpha} \approx 1.13$ for $\alpha=$ 0.05 . Although this calibration applies strictly to test for uni-modality $(k=1)$, we also use it to test for larger numbers of peaks, obtaining the probabilities shown in Table A1, fourth column. Models with $k=$ 1 or 2 peaks are rejected with high significance, while a model with $k=3$ peaks cannot be excluded.

\section{APPENDIX B: GEOMETRIC CUTS AND SELECTION EFFECTS}

In Section 3.2, we present the thin and thick disc fractions in our sample of old stars in the fiducial cut (ages $\tau>10 \mathrm{Gyr}$ and distances $d<2 \mathrm{kpc}$ ). This cut obviously introduces selection effects and those fractions should not be taken at face value. In this Appendix, we make a simple evaluation of the selection effects by varying the cuts and recalculating those fractions. Additionally to the fiducial cut, we select stars with $\tau>11 \mathrm{Gyr}$ and within the Solar cylinder, i.e. $\left(\left|R-R_{\odot}\right|<2 \mathrm{kpc}\right)$ and different cuts in $|z|$. Table $\mathrm{B} 1$ shows the number counts in the cross-match with APOGEE and the thin and thick disc fractions for different cut combinations. In general, thin disc stars correspond to $\sim 20-30$ per cent of the samples (with slightly larger or smaller fractions for cuts intended to suppress or increase the thin disc contribution), while the thick disc corresponds to $\sim 40-55$ per cent. Regardless of exact values, this indicates that the thin disc represents a significant component of the old MW.

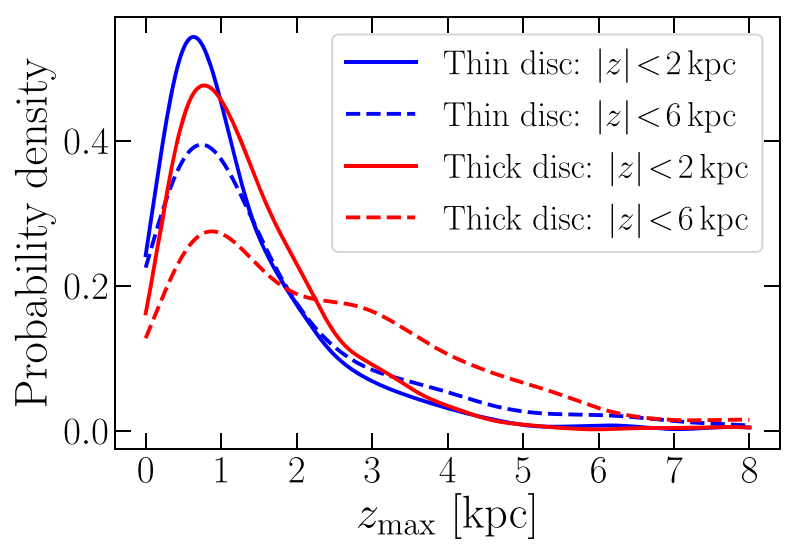

Figure B1. KDE of $z_{\max }$ for old stars in the thin and thick discs (blue and red, respectively) in the cross-match with APOGEE-DR16 within cylindrical regions around the Sun $\left(R-R_{\odot}<2 \mathrm{kpc}\right)-$ see Table B1 for details. While the thin disc KDE is changed for the different cuts, the thick disc extends to much larger $z_{\max }$ when we select stars with $|z|<6 \mathrm{kpc}$. However, even in this case, a significant fraction of chemical thick disc stars are confined to the geometric thin disc, i.e. have low $z_{\max }$.

Table B1. Simple estimates of selection effects in the cross-match with APOGEE-DR16:

\begin{tabular}{|c|c|c|c|c|c|c|}
\hline $\begin{array}{l}\tau \\
(\mathrm{Gyr})\end{array}$ & $\underset{(\mathrm{kpc})}{d}$ & $\begin{array}{c}\left|R-R_{\odot}\right| \\
(\mathrm{kpc})\end{array}$ & $\begin{array}{c}|z| \\
(\mathrm{kpc})\end{array}$ & $N$ & $\begin{array}{c}\text { Thick } \\
\text { (per cent) }\end{array}$ & $\begin{array}{c}\text { Thin } \\
\text { (per cent) }\end{array}$ \\
\hline & $<0.5$ & - & - & 222 & 40 & 50 \\
\hline & $<2$ & - & - & 1049 & 54 & 29 \\
\hline & & & $<2$ & 1489 & 52 & 26 \\
\hline \multirow[t]{6}{*}{$>10$} & - & $<2$ & $<6$ & 2238 & 46 & 17 \\
\hline & & & $0.3<|z|<6$ & 1909 & 48 & 12 \\
\hline & & & $<0.3$ & 330 & 37 & 54 \\
\hline & $<0.5$ & - & - & 124 & 46 & 37 \\
\hline & $<2$ & - & - & 573 & 54 & 25 \\
\hline & & & $<2$ & 759 & 48 & 25 \\
\hline \multirow[t]{3}{*}{$>11$} & - & $<2$ & $<6$ & 1036 & 41 & 18 \\
\hline & & & $0.3<|z|<6$ & 849 & 41 & 12 \\
\hline & & & $<0.3$ & 187 & 38 & 48 \\
\hline
\end{tabular}

Notes. For different age and geometric cuts, the right-hand columns show the fractions of thin and thick disc stars compared to the total sample (including halo stars, not shown). The fiducial cut for $\tau>10 \mathrm{Gyr}$, and the corresponding one with $\tau>11 \mathrm{Gyr}$, are shown in boldface. Neglecting cuts explicitly intended to suppress the thin disc (red), this component contribution is $\gtrsim 20$ per cent of the star counts.

Another finding of this paper is the significant fraction of chemical thick disc stars in the geometric thin disc region, i.e. at small $z_{\max }$. This could, in principle, be flawed by the suppression of thick disc stars with large $z_{\max }$ in the fiducial cut. Fig. B1 shows that the thick disc indeed extends to high $z_{\max }$ when selecting stars with $|z|<$ $6 \mathrm{kpc}$ (dashed red curve). However, its distribution still peaks at $z_{\max } \sim 1 \mathrm{kpc}$ and has a significant low $z_{\max }$ population.

This paper has been typeset from a $\mathrm{T}_{\mathrm{E}} \mathrm{X} / \mathrm{L} \mathrm{T}_{\mathrm{E}} \mathrm{X}$ file prepared by the author. 